\title{
A MULTIDIMENSIONAL SZEMERÉDI THEOREM FOR HARDY SEQUENCES OF DIFFERENT GROWTH
}

\author{
NIKOS FRANTZIKINAKIS
}

\begin{abstract}
We prove a variant of the multidimensional polynomial Szemerédi theorem of Bergelson and Leibman where one replaces polynomial sequences with other sparse sequences defined by functions that belong to some Hardy field and satisfy certain growth conditions. We do this by studying the limiting behavior of the corresponding multiple ergodic averages and obtaining a simple limit formula. A consequence of this formula in topological dynamics shows denseness of certain orbits when the iterates are restricted to suitably chosen sparse subsequences. Another consequence is that every syndetic set of integers contains certain non-shift invariant patterns, and every finite coloring of $\mathbb{N}$, with each color class a syndetic set, contains certain polychromatic patterns, results very particular to our non-polynomial setup.
\end{abstract}

\section{INTRODUCTION}

In [19], Furstenberg gave an ergodic theoretic proof of Szemerédi's theorem on arithmetic progressions, and, using similar methods, Furstenberg and Katznelson 21] proved a multidimensional extension of Szemerédi's theorem. Later on, Bergelson and Leibman 7] gave a polynomial extension of this result, a special case which states that given any collection of polynomials $p_{1}, \ldots, p_{\ell}: \mathbb{N} \rightarrow \mathbb{Z}$, with zero constant term, and vectors $\mathbf{v}_{1}, \ldots, \mathbf{v}_{\ell} \in \mathbb{Z}^{d}$, every subset of $\mathbb{Z}^{d}$ of positive upper density contains configurations of the form

$$
\left\{\mathbf{v}, \mathbf{v}+p_{1}(n) \mathbf{v}_{1}, \ldots, \mathbf{v}+p_{\ell}(n) \mathbf{v}_{\ell}\right\}
$$

for some $\mathbf{v} \in \mathbb{Z}^{d}$ and $n \in \mathbb{N}$. In the course of proving this result they introduced and studied the limiting behavior in $L^{2}(\mu)$ of the multiple ergodic averages

$$
\frac{1}{N} \sum_{n=1}^{N} f_{1}\left(T_{1}^{p_{1}(n)} x\right) \cdots f_{\ell}\left(T_{\ell}^{p_{\ell}(n)} x\right),
$$

where $T_{1}, \ldots, T_{\ell}: X \rightarrow X$ are invertible commuting measure preserving transformations acting on some probability space $(X, \mathcal{X}, \mu)$ and $f_{1}, \ldots, f_{\ell} \in L^{\infty}(\mu)$. Their goal was to prove a multiple recurrence property, namely, that for every $A \in \mathcal{X}$ with $\mu(A)>0$ one has

$$
\liminf _{N \rightarrow \infty} \frac{1}{N} \sum_{n=1}^{N} \mu\left(A \cap T_{1}^{-p_{1}(n)} A \cap \cdots \cap T_{\ell}^{-p_{\ell}(n)} A\right)>0 .
$$

Received by the editors April 24, 2012 and, in revised form, June 20, 2013.

2010 Mathematics Subject Classification. Primary 37A45; Secondary 28D05, 05D10, 11B25.

Key words and phrases. Ergodic theory, recurrence, Hardy field, Ramsey theory, nilmanifolds. The author was partially supported by Marie Curie IRG 248008. 
From this, the combinatorial result follows via the correspondence principle of Furstenberg [19, 20]. Bergelson and Leibman managed to prove this multiple recurrence property without getting very precise information about the limit of the averages (2). Two important ingredients in their proof were an ergodic structure theorem (already present in [21]) and the coloristic counterpart of their density result, now known as polynomial van der Waerden theorem, which they proved using more elementary methods 1 The reader can find several other examples were ergodic methods were used to prove combinatorial results in the surveys [3, 4, 28, 29.

In the present article, we establish a variant of the polynomial Szemerédi theorem where one replaces the polynomials $p_{1}, \ldots, p_{\ell}$ with a collection of sparse sequences of integers defined using functions that belong to some Hardy field and satisfy certain growth conditions. For instance, we show that one can substitute the configurations in (11) with configurations of the form

$$
\left\{\mathbf{v}, \mathbf{v}+\left[n^{c_{1}}\right] \mathbf{v}_{1}, \ldots, \mathbf{v}+\left[n^{c_{\ell}}\right] \mathbf{v}_{\ell}\right\}
$$

for every choice of distinct positive non-integers $c_{1}, \ldots, c_{\ell}$. Despite the similarity of this result with the polynomial Szemerédi theorem, its proof is very different. This is mainly because we are unable to prove the corresponding coloristic result in a simple way (the only proof we know uses the density result). To circumvent this problem, we deviate from the classical methods used in [7,21, and aim to prove the needed multiple recurrence property by obtaining a complete understanding of the limiting behavior of the corresponding multiple ergodic averages. In our particular setup, we establish the explicit limit formula

$$
\lim _{N \rightarrow \infty} \frac{1}{N} \sum_{n=1}^{N} f_{1}\left(T_{1}^{\left[n^{c_{1}}\right]} x\right) \cdots f_{\ell}\left(T_{\ell}^{\left[n^{\left.c_{\ell}\right]}\right.} x\right)=\tilde{f}_{1}(x) \cdots \tilde{f}_{\ell}(x),
$$

where $c_{1}, \ldots, c_{\ell}$ are distinct positive non-integers, the convergence takes place in $L^{2}(\mu)$, and $\tilde{f}_{i}$ is the orthogonal projection of the function $f_{i}$ on the subspace of functions that are left invariant by the transformation $T_{i}$. The proof of identity (4) relies on some deep decomposition results, seminorm estimates, and equidistribution results on nilmanifolds.

Because of the explicit evaluation of the limit in (4), it is a simple matter to deduce a multiple recurrence property analogous to (3), with an explicit lower bound, namely,

$$
\lim _{N \rightarrow \infty} \frac{1}{N} \sum_{n=1}^{N} \mu\left(A \cap T_{1}^{-\left[n^{c_{1}}\right]} A \cap \cdots \cap T_{\ell}^{-\left[n^{\left.c_{\ell}\right]}\right.} A\right) \geq(\mu(A))^{\ell+1},
$$

where, as usual, $c_{1}, \ldots, c_{\ell}$ are distinct positive non-integers.

We remark that identity (41) and estimate (5) fail if one of the numbers $c_{1}, \ldots, c_{\ell}$ is an integer greater than 1 . This is a known feature of polynomial sequences due to their lack of equidistribution in congruence classes. In this respect, fractional powers, as well as other sequences that we consider next, are better suited for obtaining sharp multiple recurrence results.

The method of proof of (44) allows us to work in a much more general setup. We prove that the place of the sequences $\left[n^{c_{1}}\right], \ldots,\left[n^{c_{\ell}}\right]$ can take any collection

\footnotetext{
${ }^{1}$ When $T_{1}=\cdots=T_{\ell}$, using deep results from 25, 26, 31, 36, property (3) was proved in 8. without appealing to the polynomial van der Waerden theorem. No such proof for general commuting transformations is known.
} 
of sequences $\left[a_{1}(n)\right], \ldots,\left[a_{\ell}(n)\right]$, where the functions $a_{1}(t), \ldots, a_{\ell}(t)$ belong to the same Hardy field, have different growth rates, and, roughly speaking, grow like a fractional power of $t$ (for the exact statements see Theorems 2.3 and 2.4). For instance, we can use the collection of sequences

$$
\left\{\left[n^{c}(\log n)^{d_{1}}\right], \ldots,\left[n^{c}(\log n)^{d_{\ell}}\right]\right\},
$$

where $c$ is a positive non-integer and $d_{1}, \ldots, d_{\ell} \in \mathbb{R}$ are distinct, or more exotic collections like

$$
\left\{[\sqrt[3]{n}],\left[n \sqrt{n^{3}+1}\right],\left[n^{3 / 2} e^{\sqrt{\log \log n}}\right],\left[n^{\pi} / \log n\right],\left[\sqrt{n} \int_{0}^{n} e^{\sqrt{\log t}} d t\right]\right\} .
$$

Another interesting consequence of the limit formula (4) is in topological dynamics. It enables us to show, for instance, that if $T, S$ are commuting minimal transformations acting on a compact metric space $(X, d)$, and $a, b$ are distinct positive non-integers, then for a residual set of $x \in X$ one has

$$
\overline{\left(\left(T^{\left[n^{a}\right]} x, S^{\left[n^{b}\right]} x\right)\right)_{n \in \mathbb{N}}}=X \times X .
$$

Periodic systems show that this fails if either $a$ or $b$ is an integer greater than 1 .

Lastly, the limit formula (4) gives some rather unusual consequences in combinatorics. It implies that if $E \subset \mathbb{N}$ is syndetic (i.e. has bounded gaps), then $E$ contains certain non-shift invariant patterns. For instance, we prove that for $a, b$ as before, the system

$$
\begin{aligned}
& 2 y-x=\left[n^{a}\right], \\
& 3 z-x=\left[n^{b}\right]
\end{aligned}
$$

has a solution with $x, y, z \in E$ and $n \in \mathbb{N}$. It also implies that for every finite coloring of $\mathbb{N}$, where each color class is a syndetic set, the system

$$
\begin{aligned}
& y-x=\left[n^{a}\right], \\
& z-x=\left[n^{b}\right]
\end{aligned}
$$

has a solution with $x, y, z$ having predetermined arbitrary colors. Again, these results are very particular to our non-polynomial setup and fail if either $a$ or $b$ is an integer greater than 1 .

In the next section we give a precise formulation of our main results.

\section{MAin REsults}

2.1. Our setup. In order to properly state our results we have to first introduce some notation.

A system $\left(X, \mathcal{X}, \mu, T_{1}, \ldots, T_{\ell}\right)$ is a Lebesgue probability space $(X, \mathcal{X}, \mu)$ together with a collection of commuting invertible measure preserving transformations $T_{1}, \ldots, T_{\ell}: X \rightarrow X$. By $\mathbb{E}\left(f \mid \mathcal{I}_{T_{i}}\right)$ we denote the conditional expectation on the $\sigma$-algebra $\mathcal{I}_{T_{i}}$ of $T_{i}$-invariant sets. Equivalently, this is the orthogonal projection on the closed subspace of $T_{i}$-invariant functions.

Throughout the article we use the symbol $\mathcal{H}$ to denote a translation invariant Hardy field (all notions are defined in Section 3.1). All iterates of the transformations involved in our statements are defined using functions that belong to the same Hardy field. This particular setup enables us to work within a rich class of functions and offers several aesthetic and technical advantages. 
In most instances, we restrict our attention to the following "good" class of functions:

Definition 2.1. We denote by $\mathcal{G}$ the collection of all functions $a:[c, \infty) \rightarrow \mathbb{R}$ that satisfy the growth conditions $|a(t)| /\left(t^{d} \log t\right) \rightarrow \infty$ and $|a(t)| / t^{d+1} \rightarrow 0$ as $t \rightarrow \infty$ for some integer $d \geq 0$.

The presence of the logarithm on the first condition is purely for technical reasons. It ensures that successive differences of functions in $\mathcal{G} \cap \mathcal{H}$ either converge to 0 or else are functions with substantial growth (this follows from Lemma 3.2). The key features of functions in $\mathcal{G}$ are: $(i)$ they do not grow very fast, and ( $i i)$ they "stay away" from all polynomials in a rather strong sense. Staying away from polynomials is a property that we desire since the conclusions of our main results fail for some polynomials with integer coefficients.

2.2. Results in ergodic theory. For the sake of brevity we define:

Definition 2.2. The functions $a_{1}, \ldots, a_{\ell}:[c, \infty) \rightarrow \mathbb{R}$ are said to have different growth rates if their pairwise quotients converge to $\pm \infty$ or to 0 .

2.2.1. The limit formula. The main result of this article is the following limit formula (a special case of this was stated as Problem 6 in [16] and as Problem 29 in [17]):

Theorem 2.3. Let $\mathcal{H}$ be a Hardy field and $a_{1}, \ldots, a_{\ell} \in \mathcal{G} \cap \mathcal{H}$ be functions with different growth rates. Then for every system $\left(X, \mathcal{X}, \mu, T_{1}, \ldots, T_{\ell}\right)$ and functions $f_{1}, \ldots, f_{\ell} \in L^{\infty}(\mu)$ we have

$$
\lim _{N \rightarrow \infty} \frac{1}{N} \sum_{n=1}^{N} T_{1}^{\left[a_{1}(n)\right]} f_{1} \cdots T_{\ell}^{\left[a_{\ell}(n)\right]} f_{\ell}=\tilde{f}_{1} \cdots \tilde{f}_{\ell},
$$

where $\tilde{f}_{i}:=\mathbb{E}\left(f_{i} \mid \mathcal{I}_{T_{i}}\right)=\lim _{N \rightarrow \infty} \frac{1}{N} \sum_{n=1}^{N} T_{i}^{n} f_{i}$ and the convergence takes place in $L^{2}(\mu)$.

The case $\ell=1$ follows from the equidistribution results in [10], and the case where all the iterates have sub-linear growth follows from [16] (this case is simple and no commutativity of the transformations is needed). When all the transformations are equal a slightly weaker result is proved in [16.2 Easy examples of rational rotations on the circle show that for $\ell \geq 2$ the limit formula (6) fails when the iterates are given by polynomial sequences, even if these polynomials have distinct degrees. In fact, it fails if some non-trivial linear combination of the functions $a_{1}, \ldots, a_{\ell}$ is a polynomial different than $\pm t+c$. When the assumption that the transformations commute is removed, and two or more iterates have super-linear growth, examples from [18] show that the limit in (66) does not in general exist. Lastly, we remark that in (6) the $\operatorname{limit} \lim _{N \rightarrow \infty} \frac{1}{N} \sum_{n=1}^{N}$ cannot be replaced by the uniform $\operatorname{limit} \lim _{N-M \rightarrow \infty} \frac{1}{N-M} \sum_{n=M}^{N}$. This is because for $a \in \mathcal{H} \cap \mathcal{G}$ one can show that the sequence $([a(n)])$ takes odd (respectively even) values in arbitrarily long intervals of integers.

\footnotetext{
${ }^{2}$ Even in the case where all the transformations are equal, our present argument has a technical advantage over the argument used in [16. This enables us to relax the growth condition used there.
} 
2.2.2. Multiple recurrence. Using Theorem 2.3 we easily deduce the following:

Theorem 2.4. Under the assumptions of Theorem 2.3. if $A_{0}, A_{1}, \ldots, A_{\ell} \in \mathcal{X}$ satisfy

$$
\mu\left(A_{0} \cap T_{1}^{k_{1}} A_{1} \cap \cdots \cap T_{\ell}^{k_{\ell}} A_{\ell}\right)=\alpha>0
$$

for some $k_{1}, \ldots, k_{\ell} \in \mathbb{Z}$, then

$$
\lim _{N \rightarrow \infty} \frac{1}{N} \sum_{n=1}^{N} \mu\left(A_{0} \cap T_{1}^{-\left[a_{1}(n)\right]} A_{1} \cap \cdots \cap T_{\ell}^{-\left[a_{\ell}(n)\right]} A_{\ell}\right) \geq \alpha^{\ell+1} .
$$

Proof. By Theorem 2.3 it suffices to show that

$$
\int \mathbf{1}_{A_{0}} \cdot \mathbb{E}\left(\mathbf{1}_{A_{1}} \mid \mathcal{I}_{T_{1}}\right) \cdots \mathbb{E}\left(\mathbf{1}_{A_{\ell}} \mid \mathcal{I}_{T_{\ell}}\right) d \mu \geq a^{\ell+1} .
$$

Since each function $\mathbb{E}\left(\mathbf{1}_{A_{i}} \mid \mathcal{I}_{T_{i}}\right)$ is $T_{i}$-invariant, the left hand side is greater than or equal to

$$
\int f \cdot \mathbb{E}\left(f \mid \mathcal{I}_{T_{1}}\right) \cdots \mathbb{E}\left(f \mid \mathcal{I}_{T_{\ell}}\right) d \mu \geq\left(\int f d \mu\right)^{\ell+1}=a^{\ell+1},
$$

where $f=\mathbf{1}_{A_{0} \cap T_{1}^{k_{1}} A_{1} \cap \cdots \cap T_{\ell}^{k_{\ell}} A_{\ell}}$ and the last estimate follows from Lemma 1.6 in 13 .

It follows that the limit in (7) is positive if $\mu\left(A_{0}\right)>0$ and $\mu\left(\bigcup_{k \in \mathbb{Z}} T_{i}^{k} A_{i}\right)=1$ for $i=1, \ldots, \ell$.

Applying Theorem 2.4 for $A_{0}=\cdots=A_{\ell}=A$ and $k_{1}=\cdots=k_{\ell}=0$ we deduce:

Corollary 2.5. Under the assumptions of Theorem 2.3, for every set $A \in \mathcal{X}$ we have

$$
\lim _{N \rightarrow \infty} \frac{1}{N} \sum_{n=1}^{N} \mu\left(A \cap T_{1}^{-\left[a_{1}(n)\right]} A \cap \cdots \cap T_{\ell}^{-\left[a_{\ell}(n)\right]} A\right) \geq(\mu(A))^{\ell+1} .
$$

Comments similar to those made after the statement of Theorem 2.3 apply here too. Furthermore, if $\ell=2$ and $a_{1}=a_{2}$, then no power of $\mu(A)$ can be used as a lower bound in (8) (see Theorem 2.1 in [6]).

2.3. Results in topological dynamics and combinatorics. Let $(X, d)$ be a compact metric space and $T_{1}, \ldots, T_{\ell}: X \rightarrow X$ be invertible commuting continuous transformations. There exists a Borel measure that is left invariant by all transformations. If in addition every transformation is minimal (i.e. $\overline{\left(T_{i}^{n} x\right)_{n \in \mathbb{N}}}=X$ for every $x \in X)$, then this measure gives positive value to every non-empty open set, and for every $x \in X$ and non-empty open set $U$, the set $\left\{n \in \mathbb{N}: T_{i}^{n} x \in U\right\}$ has bounded gaps (see for example [20]). As a consequence, for every $x \in X$ and nonempty open set $U$ we have $\lim _{N \rightarrow \infty} \frac{1}{N} \sum_{n=1}^{N} \mathbf{1}_{U}\left(T_{i}^{n} x\right)>0$, and using Theorem 2.3 we get for almost every $x \in X$ (and hence for a dense set of $x \in X$ ) that

$$
\limsup _{N \rightarrow \infty} \frac{1}{N} \sum_{n=1}^{N} \mathbf{1}_{U_{1}}\left(T_{1}^{\left[a_{1}(n)\right]} x\right) \cdots \mathbf{1}_{U_{\ell}}\left(T_{\ell}^{\left[a_{\ell}(n)\right]} x\right)>0
$$

whenever the sets $U_{1}, \ldots, U_{\ell}$ are taken from a given countable basis of non-empty open sets. Using this, we deduce the following (the set of $x \in X$ for which (9) holds is trivially $G_{\delta}$ and $T_{i}$-invariant). 
Theorem 2.6. Let $\mathcal{H}$ be a Hardy field and $a_{1}, \ldots, a_{\ell} \in \mathcal{G} \cap \mathcal{H}$ be functions with different growth rates. Let $(X, d)$ be a compact metric space and $T_{1}, \ldots, T_{\ell}: X \rightarrow X$ be invertible commuting minimal transformations. Then for a residual and $T_{i}$ invariant set of $x \in X$ we have

$$
\overline{\left\{\left(T_{1}^{\left[a_{1}(n)\right]} x, \ldots, T_{\ell}^{\left[a_{\ell}(n)\right]} x\right): n \in \mathbb{N}\right\}}=X \times \cdots \times X .
$$

Examples in [33. show that even when $\ell=1$ identity (9) may fail for an uncountable set of $x \in X$. In fact, for every sequence of integers $(a(n))$ with range a zero density set, it is shown in 33 that there exists a totally minimal and uniquely ergodic topological dynamical system $(X, d, T)$ such that for an uncountable set of $x \in X$ one has $x \notin \overline{\left\{T^{a(n)} x, n \in \mathbb{N}\right\}}$. Examples of minimal rotations on finite cyclic groups show that if $p \in \mathbb{Z}[t]$ is any polynomial $\neq \pm t+c$, then one may have $\overline{\left\{T^{p(n)} x, n \in \mathbb{N}\right\}} \neq X$ for every $x \in X$.

Every continuous transformation $T$ on a compact metric space $(X, d)$ has a nonempty closed $T$-invariant set $Y \subset X$ such that the transformation $T: Y \rightarrow Y$ is minimal (see for example [20]). Using this, and Theorem 2.6 for $T_{1}=\cdots=T_{\ell}=T$, we deduce:

Corollary 2.7. Let $\mathcal{H}$ be a Hardy field and $a_{1}, \ldots, a_{\ell} \in \mathcal{G} \cap \mathcal{H}$ be functions with different growth rates. Let $(X, d)$ be a compact metric space and $T: X \rightarrow X$ be an invertible continuous transformation. Then for a non-empty and T-invariant set of $x \in X$ we have

$$
\overline{\left\{\left(T^{\left[a_{1}(n)\right]} x, \ldots, T^{\left[a_{\ell}(n)\right]} x\right): n \in \mathbb{N}\right\}}=\overline{\left\{T^{n} x: n \in \mathbb{N}\right\}} \times \cdots \times \overline{\left\{T^{n} x: n \in \mathbb{N}\right\}} .
$$

Again, simple examples show this result fails if $\ell=1$ and $p \in \mathbb{Z}[t]$ is any polynomial $\neq \pm t+c$.

2.4. Combinatorial consequences. For a set $\Lambda \subset \mathbb{Z}^{d}$, we define its upper density by $\bar{d}(\Lambda):=\lim \sup _{N \rightarrow \infty}\left|\Lambda \cap[-N, N]^{d}\right| /(2 N)^{d}$ (any other shift invariant mean works for our purposes). Combining the previous multiple recurrence result with a multidimensional version of Furstenberg's correspondence principle [20, we deduce the following consequence in combinatorics:

Theorem 2.8. Let $\mathcal{H}$ be a Hardy field, $a_{1}, \ldots, a_{\ell} \in \mathcal{G} \cap \mathcal{H}$ be functions with different growth rates, and $\mathbf{v}_{1}, \ldots, \mathbf{v}_{\ell} \in \mathbb{Z}^{d}$ be vectors. Suppose that the sets $E_{0}, E_{1}, \ldots, E_{\ell} \subset$ $\mathbb{Z}^{d}$ satisfy

$$
\bar{d}\left(E_{0} \cap\left(E_{1}+k_{1}\right) \cap \cdots \cap\left(E_{\ell}+k_{\ell}\right)\right)=\alpha>0
$$

for some $k_{1}, \ldots, k_{\ell} \in \mathbb{Z}$. Then

$$
\liminf _{N \rightarrow \infty} \frac{1}{N} \sum_{n=1}^{N} \bar{d}\left(E_{0} \cap\left(E_{1}-\left[a_{1}(n)\right] \mathbf{v}_{1}\right) \cap \cdots \cap\left(E_{\ell}-\left[a_{\ell}(n)\right] \mathbf{v}_{\ell}\right)\right) \geq \alpha^{\ell+1} .
$$

Using this for $E_{0}=\cdots=E_{\ell}=E$ and $k_{1}=\cdots=k_{\ell}=0$, we get the following strengthening of the combinatorial result advertised in the introduction:

Corollary 2.9. Let $\mathcal{H}$ be a Hardy field, $a_{1}, \ldots, a_{\ell} \in \mathcal{G} \cap \mathcal{H}$ be functions with different growth rates, and $\mathbf{v}_{1}, \ldots, \mathbf{v}_{\ell} \in \mathbb{Z}^{d}$ be vectors. Then for every set $E \subset \mathbb{Z}^{d}$ we have

$$
\liminf _{N \rightarrow \infty} \frac{1}{N} \sum_{n=1}^{N} \bar{d}\left(E \cap\left(E-\left[a_{1}(n)\right] \mathbf{v}_{1}\right) \cap \cdots \cap\left(E-\left[a_{\ell}(n)\right] \mathbf{v}_{\ell}\right)\right) \geq(\bar{d}(E))^{\ell+1} \text {. }
$$


Theorem 2.8 is also applicable for syndetic sets $E_{0}, \ldots, E_{\ell} \subset \mathbb{N}$ (in this case $\alpha$ can be taken as $\left(\prod_{i=0}^{\ell} s_{i}\right)^{-1}$, where $s_{i}$ is the syndeticity constant of the set $\left.E_{i}\right)$ and gives the following:

Corollary 2.10. Let $\mathcal{H}$ be a Hardy field and $a_{1}, \ldots, a_{\ell} \in \mathcal{G} \cap \mathcal{H}$ be functions with different growth rates. Let $E_{0}, E_{1}, \ldots, E_{\ell} \subset \mathbb{N}$ be syndetic sets. Then there exist $m, n \in \mathbb{N}$ such that

$$
m \in E_{0}, m+\left[a_{1}(n)\right] \in E_{1}, \ldots, m+\left[a_{\ell}(n)\right] \in E_{\ell} .
$$

Corollary 2.10 enables us to solve some non-shift invariant systems of equations within every syndetic set. For instance, for a syndetic set $E \subset \mathbb{N}$, we can take $E_{0}:=c E, E_{i}:=c_{i} E, i=1, \ldots, \ell$, where $c, c_{i}$ are arbitrary positive integers and $c E:=\{c k, k \in E\}$, and deduce that the system of equations

$$
\begin{gathered}
c_{1} x_{1}-c x_{0}=\left[a_{1}(n)\right], \\
c_{2} x_{2}-c x_{0}=\left[a_{2}(n)\right] \\
\vdots \\
c_{\ell} x_{\ell}-c x_{0}=\left[a_{\ell}(n)\right]
\end{gathered}
$$

has a solution with $x_{0}, x_{1}, \ldots, x_{\ell} \in E$ and $n \in \mathbb{N} 3$ Another consequence is that for any finite coloring of $\mathbb{N}$, where each color class is a syndetic set, the previous system has a solution with $x_{0}, \ldots, x_{\ell}$ having arbitrary colors. In other words, if the colors classes are denoted by $C_{0}, \ldots, C_{k}$, we can have $x_{0} \in C_{i_{0}}, \ldots, x_{\ell} \in C_{i_{\ell}}$, where $i_{0}, \ldots, i_{\ell} \in\{1, \ldots, k\}$ are arbitrary.

\subsection{Key ingredients and proof plan.}

2.5.1. Key ingredients. The proof of Theorem 2.3 uses the following key ingredients:

Gowers-Host-Kra seminorms. These are non-negative numbers associated with every bounded measurable function (see Section 3.2). They were defined in a combinatorial setting in 22 and in an ergodic setting in [25]. We seek to control the $L^{2}(\mu)$ norm of the multiple ergodic averages in (6) by the seminorms of the individual functions involved.

Van der Corput's Lemma. This elementary estimate, and variations of it (see Section 3.5), is the key ingredient used to get the desired seminorm estimates.

Decomposition results. These are used to replace sequences of the form $\left(f\left(T^{n} x\right)\right)$ with sequences that have more desirable properties. We use two decompositions, one involving dual sequences (Proposition [3.4), and another, much deeper one, involving nilsequences (Theorem 3.5). Both decompositions originate from [25].

Equidistribution results on nilmanifolds. These are used towards the end of our argument when one replaces sequences of the form $\left(f\left(T^{n} x\right)\right)$ with nilsequences. They enable us to carry out the finer analysis needed to prove identity (6). The equidistribution results needed were proved in [15] using results from [23] on quantitative equidistribution of polynomial sequences on nilmanifolds.

\footnotetext{
${ }^{3}$ Similar results fail for polynomial sequences and also fail when the set $E$ is only assumed to be piecewise syndetic. Easy examples show that: (i) If $p \in \mathbb{Z}[t]$ is any polynomial different than $\pm t+c$ and $k \in \mathbb{N}$ is different than 1 , then the equation $k x-y=p(n)$ has no solution with $x, y$ belonging in some set $E$ that is an arithmetic progression. (ii) If $(a(n))$ is a sequence of integers with $a(n+1)-a(n) \rightarrow \infty$ and $k \neq 1$, then there exists a thick set $E$ such that the equation $x-k y=a(n)$ has no solution with $x, y \in E$.
} 
2.5.2. Combining the key ingredients. Crucial to the proof of Theorem 2.3 are some seminorm estimates showing that the limit in (6) is 0 when at least one of the functions involved is "uniform enough". We establish these estimates in two steps. First, we prove them for the function that is associated with the fastest growing iterate (Propositions 4.1 and 5.2). This part of the proof borrows ideas from [14] in order to devise an appropriate inductive scheme (similar to the PET induction of [2]) based on successive uses of van der Corput's Lemma. Next, we use this first step, and the decomposition result of Proposition 3.4. in order to replace one of the functions with a function that (when evaluated in the orbit of the corresponding transformation) gives rise to sequences (called dual sequences) defined by a certain averaging operation. It is then possible to carry out another induction based again on successive uses of van der Corput's Lemma in order to produce seminorm estimates for the function associated with the second fastest growing iterate (Proposition 6.2) . Continuing like this, we get seminorm estimates for all the functions (Proposition 7.1).

Using the seminorm estimates and the decomposition result of Theorem 3.5, we get that the limit in (6) remains unchanged when we replace each function with a function that pointwise gives rise to nil-sequences. At this advanced point in the proof, we are in a position to apply known equidistribution results on nilmanifolds from [15] to complete the proof of Theorem 2.3 .

For technical reasons, complications arise in implementing the previous plan when one or more iterates have sub-linear growth. These complications are handled using a variant of the aforementioned seminorm estimates (Proposition 7.3) and the equidistribution results on nilmanifolds (Proposition 7.5).

Recently, a relatively simple method for proving mean convergence of the polynomial averages (2) was developed in 35] (based on ideas from [34]), but up to this point it has not been successful in giving detailed information for the limiting function. Since the precise form of the limit is the most crucial part of our main result, and is needed for applications, it seems that we are forced to carry out the more refined analysis summarized above.

2.6. Further directions. We believe that in Theorem 2.3 (and its various consequences) the restrictions we impose on the functions $a_{1}, \ldots, a_{\ell}$ can be weakened considerably. We record here a related problem (a special case of this already appears in [16, 17]):

Problem 1. Given a Hardy field $\mathcal{H}$, show that the conclusion of Theorem 2.3 holds if the functions $a_{1}, \ldots, a_{\ell} \in \mathcal{H}$ have polynomial growth rate and every non-trivial linear combination $a(t)$ of these functions satisfies $|a(t)-c p(t)| / \log t \rightarrow \infty$ for every $c \in \mathbb{R}$ and every $p \in \mathbb{Z}[t]$.

When $\ell=1$ the result follows from the equidistribution results in [10]. The problem is open even when $\ell=2$ and $T_{1}=T_{2}$.

When the sequences $a_{1}, \ldots, a_{\ell}$ are equal, the methods used in this article do not seem particularly helpful in studying the limiting behavior of the averages in (6) (mainly because the seminorm estimates we use here fail in this case). We record a related problem (a special case of this already appears in [16, 17]):

Problem 2. Let $a:[c, \infty) \rightarrow \mathbb{R}$ be a Hardy field function with polynomial growth rate that satisfies $|a(t)-c p(t)| / \log t \rightarrow \infty$ for every $c \in \mathbb{R}$ and every $p \in \mathbb{Z}[t]$. 
Show that for every system $\left(X, \mathcal{X}, \mu, T_{1}, \ldots, T_{\ell}\right)$ and functions $f_{1}, \ldots, f_{\ell} \in L^{\infty}(\mu)$ the averages

$$
\frac{1}{N} \sum_{n=1}^{N} T_{1}^{[a(n)]} f_{1} \cdots T_{\ell}^{[a(n)]} f_{\ell}
$$

converge in $L^{2}(\mu)$ and their $\operatorname{limit}_{\text {is }} \lim _{N \rightarrow \infty} \frac{1}{N} \sum_{n=1}^{N} T_{1}^{n} f_{1} \cdots T_{\ell}^{n} f_{\ell}$ (this limit exists [34]).

The case where $T_{1}, \ldots, T_{\ell}$ are powers of a single transformation was treated in [16]. For general commuting transformations, the problem is open even when $\ell=2$ and $a(t)=t^{3 / 2}$.

Regarding pointwise convergence of the averages in (6), progress has been very scarce. The case $\ell=1$ was treated in [11, but other than this, even the simplest cases remain open.

Problem 3. Let $a, b$ be distinct positive non-integers. Show that for every ergodic system $(X, \mathcal{X}, \mu, T)$ and functions $f, g \in L^{\infty}(\mu)$, we have

$$
\lim _{N \rightarrow \infty} \frac{1}{N} \sum_{n=1}^{N} f\left(T^{\left[n^{a}\right]} x\right) \cdot g\left(T^{\left[n^{b}\right]} x\right)=\int f d \mu \cdot \int g d \mu
$$

for almost every $x \in X$.

All cases where both $a$ and $b$ are greater than 1 are open.

2.7. Notational conventions. The following notation will be used throughout the article: $\mathbb{N}=\{1,2, \ldots\}, T f=f \circ T, T^{k}=T \circ \cdots \circ T, \mathbf{1}_{E}$ is the indicator function of a set $E$, and $\mathcal{C}^{k} z$ is $z$ if $k$ is even and $\bar{z}$ if $z$ is odd. We often write $\infty$ instead of $+\infty$. If $a(t), b(t)$ are real valued functions defined on some half line $[c, \infty)$, we write $a(t) \prec b(t)$ if $a(t) / b(t) \rightarrow 0$ as $t \rightarrow \infty$. We write $a(t) \ll b(t)$ if there exists $C \in \mathbb{R}$ such that $|a(t)| \leq C|b(t)|$ for all large enough $t \in \mathbb{R}$, and $a \sim b$ if $a(t) / b(t)$ converges to a non-zero constant as $t \rightarrow \infty$. We denote by $S_{h} a$ the function defined by $\left(S_{h} a\right)(t)=a(t+h)$. A function $a:[c, \infty) \rightarrow \mathbb{R}$ has degree $d$ if $t^{d} \ll a(t) \prec t^{d+1}$. By $\mathcal{H}$ we denote a translation invariant Hardy field and by $\mathcal{G}$ the set of functions $a:[c, \infty) \rightarrow \mathbb{R}$ that satisfy $t^{k} \log t \prec a(t) \prec t^{k+1}$ for some integer $k \geq 0$. If $(X, \mathcal{X}, \mu, T)$ is a system, $\mathcal{I}_{T}$ denotes the $\sigma$-algebra of $T$-invariant sets and $\mathbb{E}\left(f \mid \mathcal{I}_{T}\right)$ the corresponding conditional expectation.

\section{BACKGROUND MATERIAL}

In this section we put together some background material that we use throughout this article.

3.1. Basic facts about Hardy fields. Let $B$ be the collection of equivalence classes of real valued functions defined on some half line $[c, \infty)$, where we identify two functions if they agree eventually 4 A Hardy field $\mathcal{H}$ is a subfield of the ring $(B,+, \cdot)$ that is closed under differentiation (a term first used by the Bourbaki group in [12). A Hardy field function is a function that belongs to some Hardy field.

\footnotetext{
${ }^{4}$ The equivalence classes just defined are often called germs of functions. We choose to use the word function when we refer to elements of $B$ instead, with the understanding that all the operations defined and statements made for elements of $B$ are considered only for sufficiently large values of $t \in \mathbb{R}$.
} 
We are going to assume throughout that all Hardy fields mentioned are translation invariant, meaning that if $a(t) \in \mathcal{H}$, then $a(t+h) \in \mathcal{H}$ for every $h \in \mathbb{R}$.

A particular example of such a Hardy field is the set $\mathcal{L E}$ that was introduced by Hardy in 24] and consists of all logarithmic-exponential functions, meaning all functions defined on some half line $[c, \infty)$ by a finite combination of the symbols ,,$+- \times,:, \log$, exp, operating on the real variable $t$ and on real constants. For example, functions such as $t^{\sqrt{2}}, t(\log t)^{2}, e^{t^{2}}$, and $e^{\sqrt{\log \log t}} / \log \left(t^{2}+1\right)$ are all elements of $\mathcal{L} \mathcal{E}$. Another, even more extensive, example was constructed by Boshernitzan in [9]. It satisfies the following properties:

- it contains the set $\mathcal{L E}$;

- it is closed under integration; and

- it is closed under composition of functions that increase to infinity.

Every Hardy field function is eventually monotonic. If one of the functions $a, b:[c, \infty) \rightarrow \mathbb{R}$ belongs to a Hardy field, and the other function belongs to the same Hardy field or to $\mathcal{L} \mathcal{E}$, then the limit $\lim _{t \rightarrow \infty} a(t) / b(t)$ exists (possibly infinite). This property is key and will often justify our use of l'Hopital's rule. We are going to freely use all these properties without any further explanation in the sequel. The reader can find more information about Hardy fields in [9, 10] and the references therein.

Definition 3.1. We say that two functions $a, b:[c, \infty) \rightarrow \mathbb{R}$ have the same growth rate, and write $a \sim b$ if $a(t) / b(t)$ converges to a non-zero constant as $t \rightarrow \infty$. We say that the function $a:[c, \infty) \rightarrow \mathbb{R}$ has polynomial growth if $a(t) \prec t^{k}$ for some $k \in \mathbb{N}$.

Notice that if the functions $a, b$ belong to the same Hardy field, then one of the following three alternatives holds: $a \prec b, b \prec a, a \sim b$. A key property of Hardy field functions with polynomial growth is that we can relate their growth rates with the growth rates of their derivatives:

Lemma 3.2. Let $a:[c, \infty) \rightarrow \mathbb{R}$ be a Hardy field function with polynomial growth.

(i) If $a \succ 1$, then $a^{\prime} \ll a / t$.

(ii) If $a \succ t^{\varepsilon}$ for some $\varepsilon>0$, then $a^{\prime} \sim a / t$ and for every non-zero $h \in \mathbb{R}$ we have $S_{h} a-a \sim a / t$.

Proof. If $t a^{\prime}(t) \ll 1$, then the conclusion of part $(i)$ is obvious. Suppose that $t a^{\prime}(t) \rightarrow \infty$. Applying l'Hopital's rule we get

$$
\lim _{t \rightarrow \infty} \frac{t a^{\prime}(t)}{a(t)}=\lim _{t \rightarrow \infty} \frac{(\log |a(t)|)^{\prime}}{(\log t)^{\prime}}=\lim _{t \rightarrow \infty} \frac{\log |a(t)|}{\log t} .
$$

Since $a(t)$ has polynomial growth, the last limit is a non-negative real number. Hence, $a^{\prime} \ll a / t$.

If furthermore one has $t^{\varepsilon} \prec a(t)$ for some $\varepsilon>0$ and $a(t)$ has polynomial growth, then the previous limit is a positive real number. This implies that $a^{\prime} \sim a / t$. Lastly, suppose that $h>0$ (a similar argument applies if $h<0$ ). The mean value theorem gives that

$$
a(t+h)-a(t)=h a^{\prime}\left(\xi_{t}\right)
$$

for some $\xi_{t} \in[t, t+h]$. Applying l'Hopital's rule we get $a^{\prime}\left(\xi_{t}\right) / a^{\prime}(t) \sim a\left(\xi_{t}\right) / a(t)$ and one easily sees that $a\left(\xi_{t}\right) / a(t) \rightarrow 1$ (if not, then $\lim |a(t+h) / a(t)|>1$, contradicting the fact that $a(t)$ has polynomial growth). Combining the above we get $S_{h} a-a \sim a^{\prime}$. The proof is complete since by the first claim $a^{\prime} \sim a / t$. 
3.2. Basic facts from ergodic theory. A system $(X, \mathcal{X}, \mu, T)$ is a Lebesgue probability space $(X, \mathcal{X}, \mu)$ together with an invertible measure preserving transformations $T: X \rightarrow X$.

The ergodic theorem. The ergodic theorem states that for every system $(X, \mathcal{X}, \mu, T)$ and function $f \in L^{1}(\mu)$ we have for almost every $x \in X$ that

$$
\lim _{N \rightarrow \infty} \frac{1}{N} \sum_{n=1}^{N} f\left(T^{n} x\right)=\tilde{f}(x)
$$

where $\tilde{f}=\mathbb{E}\left(f \mid \mathcal{I}_{T}\right)$ and

$$
\mathcal{I}_{T}:=\left\{A \in \mathcal{X}: \mu\left(T^{-1} A \triangle A\right)=0\right\} .
$$

Gowers-Host-Kra uniformity seminorms. Following [25, where a similar definition was given for ergodic systems, given a system $(X, \mathcal{X}, \mu, T)$ and a function $f \in$ $L^{\infty}(\mu)$, we define inductively

$$
\begin{gathered}
\|f\|_{1, T}:=\left\|\mathbb{E}\left(f \mid \mathcal{I}_{T}\right)\right\|_{L^{2}(\mu)} \\
\|f\|_{k+1, T}^{2^{k+1}}:=\lim _{N \rightarrow \infty} \frac{1}{N} \sum_{n=1}^{N}\left\|\bar{f} \cdot T^{n} f\right\|_{k, T}^{2^{k}} .
\end{gathered}
$$

That all limits exist and $\|\cdot\|_{k, T}$ is a seminorm can be proved as in 25. Furthermore, the limit in (13) remains unchanged if replaced with the uniform limit $\lim _{N-M \rightarrow \infty} \frac{1}{N-M} \sum_{n=M}^{N-1}$. Using the ergodic theorem one gets

$$
\|f\|_{1, T}^{2}=\lim _{N \rightarrow \infty} \frac{1}{N} \sum_{n=1}^{N} \int \bar{f} \cdot T^{n} f d \mu,
$$

and more generally, that

$$
\|f\|_{k}^{2^{k}}=\lim _{N \rightarrow \infty} \frac{1}{N} \sum_{n_{k}=1}^{N} \cdots \lim _{N \rightarrow \infty} \frac{1}{N} \sum_{n_{1}=1}^{N} \int \prod_{\boldsymbol{\epsilon} \in\{0,1\}^{k}} \mathcal{C}^{|\boldsymbol{\epsilon}|} T^{\boldsymbol{\epsilon} \cdot \mathbf{n}} f d \mu,
$$

where $\mathbf{n}=\left(n_{1}, \ldots, n_{k}\right)$ and for $\boldsymbol{\epsilon} \in\{0,1\}^{k}$ we let

$$
\mathbf{n} \cdot \boldsymbol{\epsilon}:=n_{1} \epsilon_{1}+\cdots+n_{k} \epsilon_{k}, \quad|\boldsymbol{\epsilon}|=\epsilon_{1}+\cdots+\epsilon_{k},
$$

and for $z \in \mathbb{C}$ and $k$ non-negative integer we let

$$
\mathcal{C}^{k} z:= \begin{cases}z & \text { if } k \text { is even } \\ \bar{z} & \text { if } k \text { is odd. }\end{cases}
$$

It follows from Theorem 13.1 in [25] that in (14) the iterative limit can be replaced with the $\operatorname{limit}_{N \rightarrow \infty} \frac{1}{N^{k}} \sum_{1 \leq n_{1}, \ldots, n_{k} \leq N}$. Using (14) and the ergodic theorem one can check that

$$
\|f \otimes \bar{f}\|_{k, T \times T} \leq\|f\|_{k+1, T}^{2}
$$

holds for every $k \in \mathbb{N}$. We also remark that $\|f\|_{k, T} \leq\|f\|_{k+1, T}$ holds for every $k \in \mathbb{N}$. 


\subsection{Dual functions, dual sequences, and weak decomposition.}

3.3.1. Dual functions. Let $(X, \mathcal{X}, \mu, T)$ be a system, $f \in L^{\infty}(\mu)$, and $M \in \mathbb{N}$. We define

$$
A_{M}(f):=\frac{1}{M^{k}} \sum_{\mathbf{m} \in[1, M]^{k}} \prod_{\substack{\boldsymbol{\epsilon} \in\{0,1\}^{k}, \epsilon \neq 00 \cdots 0}} \mathcal{C}^{|\boldsymbol{\epsilon}|} T^{\mathbf{m} \cdot \boldsymbol{\epsilon}} f .
$$

It is shown in [25] that the averages $A_{M}(f)$ converge in $L^{2}(\mu)$, and in [1] it is also shown that they converge pointwise. We define

$$
\mathcal{D}_{k, T} f:=\lim _{M \rightarrow \infty} A_{M}(f)
$$

and call any such function a level $k$ dual function. For instance, we have

$$
\left(\mathcal{D}_{2, T} f\right)(x)=\lim _{M \rightarrow \infty} \frac{1}{M^{2}} \sum_{1 \leq m_{1}, m_{2} \leq M} T^{m_{1}} \bar{f} \cdot T^{m_{2}} \bar{f} \cdot T^{m_{1}+m_{2}} f .
$$

The importance of dual functions in the current article stems from the following result (it follows from (14) and the fact that the iterative limit can be replaced with a limit over cubes):

Proposition 3.3. Let $(X, \mathcal{X}, \mu, T)$ be a system. Then for every $f \in L^{\infty}(\mu)$ and $k \in \mathbb{N}$ we have

$$
\int f \cdot \mathcal{D}_{k, T} f d \mu=\|f\|_{k, T}^{2^{k}}
$$

As a consequence, $\|f\|_{k, T} \neq 0$ if $f$ positively correlates with some dual function of level $k$. The converse is also true.

3.3.2. Dual sequences. Adual sequence of level $k$ is a sequence $(\mathcal{D}(n))$ of the form

$$
\mathcal{D}(n):=\lim _{M \rightarrow \infty} \frac{1}{M^{k}} \sum_{\mathbf{m} \in[1, M]^{k}} \prod_{\substack{\boldsymbol{\epsilon} \in\{0,1\}^{k}, \epsilon \neq 00 \cdots 0}} \mathcal{C}^{|\boldsymbol{\epsilon}|} d(n+\mathbf{m} \cdot \boldsymbol{\epsilon}),
$$

where $(d(n))$ is a bounded sequence such that the above limit exists for every $n \in \mathbb{N}$.

For future use, we record the identity

$$
\mathcal{D}(n)=\lim _{M \rightarrow \infty} \frac{1}{M^{k}} \sum_{\mathbf{m} \in[1, M]^{k}} \prod_{\substack{\boldsymbol{\epsilon} \in\{0,1\}^{k}, \epsilon \neq 00 \cdots 0}} \mathcal{C}^{|\boldsymbol{\epsilon}|} \mathbf{d}_{\boldsymbol{\epsilon}}(\mathbf{m}+n \tilde{\boldsymbol{\epsilon}}),
$$

where $\tilde{\boldsymbol{\epsilon}}$ is any vector in $\{0,1\}^{k}$ such that $\boldsymbol{\epsilon} \cdot \tilde{\boldsymbol{\epsilon}}=1$ and

$$
\mathbf{d}_{\boldsymbol{\epsilon}}(\mathbf{m})=d(\boldsymbol{\epsilon} \cdot \mathbf{m}) \text {. }
$$

For instance, if $(\mathcal{D}(n))$ is a dual sequence of level 2 , then

$$
\begin{aligned}
\mathcal{D}(n) & =\lim _{M \rightarrow \infty} \frac{1}{M^{2}} \sum_{1 \leq m_{1}, m_{2} \leq N} \bar{d}\left(n+m_{1}\right) \cdot \bar{d}\left(n+m_{2}\right) \cdot d\left(n+m_{1}+m_{2}\right) \\
& =\lim _{M \rightarrow \infty} \frac{1}{M^{2}} \sum_{1 \leq m_{1}, m_{2} \leq N} \overline{\mathbf{d}}_{1}\left(m_{1}+n, m_{2}\right) \cdot \overline{\mathbf{d}}_{2}\left(m_{1}, m_{2}+n\right) \cdot \mathbf{d}_{3}\left(m_{1}+n, m_{2}\right),
\end{aligned}
$$

where

$$
\mathbf{d}_{1}\left(m_{1}, m_{2}\right):=d\left(m_{1}\right), \quad \mathbf{d}_{1}\left(m_{1}, m_{2}\right):=d\left(m_{2}\right), \quad \mathbf{d}_{3}\left(m_{1}, m_{2}\right):=d\left(m_{1}+m_{2}\right) .
$$


3.3.3. Weak decomposition. For the purpose of this article the significance of the collection of dual sequences stems from the following decomposition result:

Proposition 3.4 (Weak decomposition). Let $(X, \mathcal{X}, \mu, T)$ be a system, $f \in L^{\infty}(\mu)$, and $k \in \mathbb{N}$. Then for every $\varepsilon>0$, there exist functions $f_{s}, f_{u}, f_{e} \in L^{\infty}(\mu)$, such that

(1) $f=f_{s}+f_{u}+f_{e}$;

(2) $\left\|f_{u}\right\|_{k}=0 ;\left\|f_{e}\right\|_{L^{1}(\mu)} \leq \varepsilon ;$ and

(3) $f_{s}=\sum_{i=1}^{m} c_{i} f_{s, i}$, where $c_{i} \in \mathbb{R}, f_{s, i} \in L^{\infty}(\mu)$, and for almost every $x \in X$ the sequence $\left(f_{s, i}\left(T^{n} x\right)\right)_{n \in \mathbb{N}}$ is a dual sequence of level $k$.

Proof. Let $\varepsilon>0, k \in \mathbb{N}$, and $f \in L^{\infty}(\mu)$. We construct an invariant sub- $\sigma$-algebra $\mathcal{Z}_{k-1}$ of $\mathcal{X}$ exactly as in Section 4 of [25]. It satisfies the same property as in Lemma 4.3 of [25], namely,

$$
\text { for } f \in L^{\infty}(\mu), \mathbb{E}\left(f \mid \mathcal{Z}_{k-1}\right)=0 \text { if and only if }\|f\|_{k}=0 \text {. }
$$

We can decompose $f$ as $f=f_{u}+g$, where $g=\mathbb{E}\left(f \mid \mathcal{Z}_{k-1}\right)$ and $f_{u} \perp L^{\infty}\left(\mathcal{Z}_{k-1}, \mu\right)$. It follows from (17) that $\left\|f_{u}\right\|_{k}=0$. It is clear that $f_{u}, g, \in L^{\infty}(\mu)$.

We claim that linear combinations of dual functions of level $k$ are dense in $L^{1}\left(\mathcal{Z}_{k-1}, \mu\right)$. Indeed, by duality, it suffices to show that if $\tilde{f} \in L^{\infty}\left(\mathcal{Z}_{k-1}, \mu\right)$ satisfies $\int \tilde{f} \cdot \mathcal{D}_{k, T} f d \mu=0$ for every $f \in L^{\infty}(\mu)$, then $\tilde{f}=0$. Taking $f=\tilde{f}$ gives $\int \tilde{f} \cdot \mathcal{D}_{k, T} \tilde{f} d \mu=0$, and by Proposition 3.3 we get $\|\tilde{f}\|_{k}=0$. Since $\tilde{f} \in L^{\infty}\left(\mathcal{Z}_{k-1}, \mu\right)$, we deduce from (17) that $\tilde{f}=0$. This completes the proof of the claim.

Keeping in mind that $g \in L^{\infty}\left(\mathcal{Z}_{k-1}, \mu\right)$, the claim enables us to decompose $g$ as $g=f_{s}+f_{e}$, where $f_{s}$ is a finite linear combination of dual functions of level $k$ and $\left\|f_{e}\right\|_{L^{1}(\mu)} \leq \varepsilon$. Since the function $g$ and all dual functions are bounded, the function $f_{e}$ is bounded. The proof ends upon noticing that if $h$ is a dual function of level $k$, then for almost every $x \in X$ the sequence $\left(h\left(T^{n} x\right)\right)_{n \in \mathbb{N}}$ is a dual sequence of level $k$.

3.4. Nilsystems, nilsequences, and strong decomposition. A nilmanifold is a homogeneous space $X=G / \Gamma$, where $G$ is a nilpotent Lie group and $\Gamma$ is a discrete cocompact subgroup of $G$. If $G_{k+1}=\{e\}$, where $G_{k}$ denotes the $k$-th commutator subgroup of $G$, we say that $X$ is a $k$-step nilmanifold.

A $k$-step nilpotent Lie group $G$ acts on $G / \Gamma$ by left translation where the translation by a fixed element $a \in G$ is given by $T_{a}(g \Gamma)=(a g) \Gamma$. By $m_{X}$ we denote the unique probability measure on $X$ that is invariant under the action of $G$ by left translation (called the normalized Haar measure), and by $\mathcal{G} / \Gamma$ we denote the Borel $\sigma$-algebra of $G / \Gamma$. Fixing an element $a \in G$, we call the system $\left(G / \Gamma, \mathcal{G} / \Gamma, m_{X}, T_{a}\right)$ a $k$-step nilsystem. The reader can find more material about nilmanifolds in 31 . and the references therein.

If $X=G / \Gamma$ is a $k$-step nilmanifold, $a \in G, x \in X$, and $f \in C(X)$, we call the sequence $\left(f\left(a^{n} x\right)\right)_{n \in \mathbb{N}}$ a basic $k$-step nilsequence. A $k$-step nilsequence is a uniform limit of basic $k$-step nilsequences.

3.4.1. Strong decomposition. The next decomposition result will be crucial for our study. For ergodic systems it is a direct consequence of a deep structure theorem in [25]; the extension to the non-ergodic case was treated in [14] (see Proposition 3.1). 
Theorem 3.5 (Strong decomposition). Let $(X, \mathcal{X}, \mu, T)$ be a system, $f \in L^{\infty}(\mu)$, and $k \in \mathbb{N}$. Then for every $\varepsilon>0$, there exist functions $f_{s}, f_{u}, f_{e} \in L^{\infty}(\mu)$, with $L^{\infty}(\mu)$ norm at most $2\|f\|_{L^{\infty}(\mu)}$, such that

(1) $f=f_{s}+f_{u}+f_{e}$;

(2) $\left\|f_{u}\right\|_{k+1}=0 ;\left\|f_{e}\right\|_{L^{2}(\mu)} \leq \varepsilon ;$ and

(3) for almost every $x \in X$ the sequence $\left(f_{s}\left(T^{n} x\right)\right)_{n \in \mathbb{N}}$ is a $k$-step nilsequence.

3.5. The van der Corput Lemma. A key tool in proving uniformity estimates is the following variant of van der Corput's fundamental estimate (for a proof see [2] or Lemma 3.1 in [30]):

Lemma 3.6. Let $N \in \mathbb{N}$ and $v_{1}, \ldots, v_{N}$ be vectors in an inner product space. Then for every integer $H$ between 1 and $N$ we have $(\Re(z)$ denotes the real part of a complex number $z$ )

$$
\left\|\frac{1}{N} \sum_{n=1}^{N} v_{n}\right\|^{2} \leq \frac{2}{H} \sum_{h=1}^{H}\left(1-\frac{h}{H}\right) \Re\left(\frac{1}{N} \sum_{n=1}^{N}\left\langle v_{n+h}, v_{n}\right\rangle\right)+\frac{2}{H}+\frac{4 H}{N} .
$$

We also use the following qualitative variant:

Lemma 3.7. Let $\left(v_{n}\right)$ be a bounded sequence of vectors in an inner product space, and $\left(\Phi_{N}\right)$ be a Følner sequence of subsets of $\mathbb{N}$. Then

$$
\limsup _{N \rightarrow \infty}\left\|\frac{1}{\left|\Phi_{N}\right|} \sum_{n \in \Phi_{N}} v_{n}\right\|^{2} \leq 4 \limsup _{H \rightarrow \infty} \frac{1}{H} \sum_{h=1}^{H} \limsup _{N \rightarrow \infty}\left|\frac{1}{\left|\Phi_{N}\right|} \sum_{n \in \Phi_{N}}\left\langle v_{n+h}, v_{n}\right\rangle\right| .
$$

In most cases we apply this lemma for $\Phi_{N}=[1, N], N \in \mathbb{N}$.

\section{Seminorm estimates for the highest Degree iterate: TWO TRANSFORMATIONS}

An important step towards establishing Theorem 2.3 is to obtain estimates that enable us to control the $L^{2}(\mu)$ norm of the averages in (6) by the uniformity seminorms of the individual functions. In this section and the next one, our goal is to do this for the function that is associated with the fastest growing iterate. In subsequent sections we utilize this information in order to get similar estimates for the other functions.

Since the proof is notationally heavy, we choose to first present it in detail for the case of two commuting transformations. The argument that covers the general case is very similar, and we sketch its proof in the next section.

The main goal in this section is to establish the following result:

Proposition 4.1. Let $\left(X, \mathcal{X}, \mu, T_{1}, T_{2}\right)$ be a system and $f_{1}, f_{2} \in L^{\infty}(\mu)$ be functions. Let $\mathcal{H}$ be a Hardy field, $a_{1}, a_{2} \in \mathcal{G} \cap \mathcal{H}$ be functions that satisfy $a_{1} \succ a_{2}$, and let $d:=\operatorname{deg}\left(a_{1}\right)$ (all notions are defined in Section 4.1). Then there exists $k=k(d)$ such that: If $\left\|f_{1}\right\|_{k, T_{1}}=0$, then the averages

$$
\frac{1}{N} \sum_{n=1}^{N} T_{1}^{\left[a_{1}(n)\right]} f_{1} \cdot T_{2}^{\left[a_{2}(n)\right]} f_{2}
$$

converge to 0 in $L^{2}(\mu)$. 
Our method necessitates that we prove a more general result that we present next.

Proposition 4.2. Let $\left(X, \mathcal{X}, \mu, T_{1}, T_{2}\right)$ be a system and $f_{1}, \ldots, f_{m} \in L^{\infty}(\mu)$ be functions. Let $(\mathcal{A}, \mathcal{B})$ be a nice ordered family of pairs of functions with degree $d$ (all notions are defined in Sections 4.1 and 4.2). Then there exists $k=k(d, m) \in \mathbb{N}$ such that: If $\left\|f_{1}\right\|_{k, T_{1}}=0$, then

$$
\lim _{N \rightarrow \infty} \sup _{E \subset \mathbb{N}}\left\|\frac{1}{N} \sum_{n=1}^{N} \prod_{i=1}^{m} T_{1}^{\left[a_{i}(n)\right]} T_{2}^{\left[b_{i}(n)\right]} f_{i} \cdot \mathbf{1}_{E}(n)\right\|_{L^{2}(\mu)}=0 .
$$

Applying this result to the nice family $(\mathcal{A}, \mathcal{B})$ defined by $\mathcal{A}:=\left(a_{1}, 0\right)$ and $\mathcal{B}:=$ $\left(0, a_{2}\right)$, one sees that Proposition 4.1 follows from Proposition 4.2 .

\subsection{Families of pairs of functions and their type.}

\subsubsection{Degree and equivalence.}

Definition 4.3. If $a:[c, \infty) \rightarrow \mathbb{R}$ is a function with polynomial growth rate, and $k_{0}$ is the smallest non-negative integer $k$ such that $a(t) \prec t^{k}$, we define $d:=k_{0}-1$ to be the degree of the function, and write $\operatorname{deg}(a)=d$.

If $a(t)$ is a Hardy field function, then $\operatorname{deg}(a)=-1$ if and only if $a(t) \rightarrow 0$, and $\operatorname{deg}(a)=d \geq 0$ if and only if $t^{d} \ll a(t) \prec t^{d+1}$. For example, $\operatorname{deg}(1 / t)=-1$, $\operatorname{deg}(1)=\operatorname{deg}(\sqrt{t})=\operatorname{deg}(t / \log t)=0, \operatorname{deg}(t)=\operatorname{deg}\left(t^{1.5}\right)=1$.

We remind the reader that two functions $a, b:[c, \infty) \rightarrow \mathbb{R}$ have the same growth rate, in which case we write $a \sim b$ if $a(t) / b(t)$ converges to a non-zero constant as $t \rightarrow \infty$. We will make use of the following stronger notion of growth equivalence:

Definition 4.4. We say that two functions $a, b:[c, \infty) \rightarrow \mathbb{R}$ are equivalent, and write $a \cong b$ if they have polynomial growth rate and satisfy

$$
\operatorname{deg}(a-b)<\min \{\operatorname{deg}(a), \operatorname{deg}(b)\} .
$$

Notice that if $a \cong b$, then $a(t) / b(t) \rightarrow 1$, but the converse is not true. For example $t^{1.5} \not t^{1.5}+t^{1.1}$.

4.1.2. Families of pairs of functions. Let $m \in \mathbb{N}$. Given two ordered families of functions

$$
\mathcal{A}:=\left(a_{1}, \ldots, a_{m}\right), \quad \mathcal{B}:=\left(b_{1}, \ldots, b_{m}\right),
$$

where $a_{i}, b_{i}:[c, \infty) \rightarrow \mathbb{R}$ have polynomial growth rate, we define the ordered family of pairs of functions $(\mathcal{A}, \mathcal{B})$ as follows:

$$
(\mathcal{A}, \mathcal{B}):=\left(\left(a_{1}, b_{1}\right), \ldots,\left(a_{m}, b_{m}\right)\right) .
$$

The reader is advised to think of this family of pairs as an efficient way to record the functions that appear in the iterates (18).

The maximum of the degrees of the functions in the families $\mathcal{A}$ and $\mathcal{B}$ is called the degree of the family $(\mathcal{A}, \mathcal{B})$.

For convenience of exposition, if pairs of bounded functions appear in $(\mathcal{A}, \mathcal{B})$ we remove them, and henceforth we assume:

All families $(\mathcal{A}, \mathcal{B})$ that we consider do not contain pairs of bounded functions. 
4.1.3. Definition of type. We fix a non-negative integer $d$ and restrict ourselves to families $(\mathcal{A}, \mathcal{B})$ with degree between 0 and $d$.

Let

$$
\mathcal{A}^{\prime}:=\{a \in \mathcal{A}: a \text { is not bounded }\}
$$

and

$$
\mathcal{B}^{\prime}:=\left\{b_{i} \in \mathcal{B}: a_{i} \text { is bounded }\right\} .
$$

For $i=0,1, \ldots, d$, let $w_{1, i}, w_{2, i}$ be the number of distinct non-equivalent classes of functions of degree $i$ in $\mathcal{A}^{\prime}$ and $\mathcal{B}^{\prime}$ correspondingly (if $\mathcal{B}^{\prime}$ is empty, then $w_{2, i}=0$ for $i=0,1, \ldots, d)$.

We define the (matrix) type of the family $(\mathcal{A}, \mathcal{B})$ to be the $2 \times(d=1)$ matrix

$$
\left(\begin{array}{lll}
w_{1, d} & \ldots & w_{1,0} \\
w_{2, d} & \ldots & w_{2,0}
\end{array}\right)
$$

For example, consider the family of pairs

$$
\left(\left(t^{2.5}, t^{3.5}\right),\left(t^{2.5}+t^{2}, t\right),\left(t^{2.5}+t^{1.5}, 2 t\right),\left(t^{0.5}, t\right)\left((t+1)^{0.5}-t^{0.5}, t^{1.5}\right),\left(0, t^{0.5}\right)\right) .
$$

Then $d=3, \mathcal{A}^{\prime}=\left\{t^{2.5}, t^{2.5}+t^{2}, t^{2.5}+t^{1.5}, t^{0.5}\right\}$, and $\mathcal{B}^{\prime}=\left\{t^{1.5}, t^{0.5}\right\}$. As a consequence, the family of pairs $(\mathcal{A}, \mathcal{B})$ has type

$$
\left(\begin{array}{llll}
0 & 2 & 0 & 1 \\
0 & 0 & 1 & 1
\end{array}\right)
$$

We order the set of all possible types lexicographically: we start by comparing the first element of the first row of each matrix, and after going through all the elements of the first row, we compare the elements of the second row of each matrix, and so on. In other words, given two $2 \times(d+1)$ matrices $W:=\left(w_{i, j}\right)$ and $W^{\prime}:=\left(w_{i, j}^{\prime}\right)$, we say that $W \succ W^{\prime}$ if: $w_{1, d}>w_{1, d}^{\prime}$, or $w_{1, d}=w_{1, d}^{\prime}$ and $w_{1, d-1}>w_{1, d-1}^{\prime}, \ldots$, or $w_{1, i}=w_{1, i}^{\prime}$ for $i=0, \ldots, d$ and $w_{2, d}>w_{2, d}^{\prime}$, and so on.

As an example we mention

$$
\left(\begin{array}{ll}
2 & 2 \\
0 & 0
\end{array}\right) \succ\left(\begin{array}{cc}
2 & 1 \\
\star & \star
\end{array}\right) \succ\left(\begin{array}{cc}
2 & 0 \\
\star & \star
\end{array}\right) \succ\left(\begin{array}{cc}
1 & \star \\
\star & \star
\end{array}\right) \succ\left(\begin{array}{cc}
0 & \star \\
\star & \star
\end{array}\right) \succeq\left(\begin{array}{cc}
0 & 0 \\
\star & \star
\end{array}\right) \succeq\left(\begin{array}{cc}
0 & 0 \\
0 & \star
\end{array}\right) \succeq\left(\begin{array}{ll}
0 & 0 \\
0 & 0
\end{array}\right),
$$

where in the place of the stars one can put any collection of non-negative integers.

An important observation is that although for a given type $W$ there is an infinite number of possible types $W^{\prime}$ that are smaller than $W$, we have

Lemma 4.5. Every decreasing sequence of types of families of pairs is eventually stationary.

Therefore, if some operation reduces the type of a certain family of pairs of functions, then after a finite number of repetitions it will terminate.

4.2. Nice families and the van der Corput operation. In this subsection we define a class of "nice" families of pairs of functions that will be instrumental for our subsequent discussion. Furthermore, we define an operation that maps nice families to nice families and reduces their type. 
4.2.1. Nice families. We remind the reader of our definition of the class of good functions:

$\mathcal{G}=\left\{a:[c, \infty) \rightarrow \mathbb{R}\right.$ such that $t^{d} \log t \prec a(t) \prec t^{d+1}$ for some integer $\left.d \geq 0\right\}$.

Definition 4.6. Given a function $a:[c, \infty) \rightarrow \mathbb{R}$, we define $\mathcal{F}(a)$ to be the family of functions that contains all integer combinations of shifts of $a$, meaning

$$
\mathcal{F}(a):=\left\{\sum_{i=1}^{l} k_{i} \cdot S_{h_{i}} a, k_{i} \in \mathbb{Z}, h_{i}, l \in \mathbb{N}\right\} .
$$

Using Lemma 3.2, one sees that if $a \in \mathcal{G}$ and $b \in \mathcal{F}(a)$, then either $b(t) \rightarrow 0$ or $b \in \mathcal{G}$ (see also Lemma 4.8).

Henceforth, we are going to work with the following class of pairs of functions:

Definition 4.7. Let $\mathcal{H}$ be a Hardy field, $a, b \in \mathcal{G} \cap \mathcal{H}, a_{i} \in \mathcal{F}(a), b_{i} \in \mathcal{F}(b)$ for $i=1, \ldots, m$, and $\mathcal{A}:=\left(a_{1}, \ldots, a_{m}\right), \mathcal{B}:=\left(b_{1}, \ldots, b_{m}\right)$. We call the ordered family $(\mathcal{A}, \mathcal{B})$ nice if

(1) $a_{1}-a_{i} \succ 1$ and $a_{i} \ll a_{1}$ for $i=2, \ldots, m$;

(2) $b_{i} \prec a_{1}$ for $i=1, \ldots, m$;

(3) $b_{1}-b_{i} \prec a_{1}-a_{i}$ for $i=2, \ldots, m$.

For example, if $\mathcal{H}$ is a Hardy field, and $a, b \in \mathcal{G} \cap \mathcal{H}$ satisfy $a \succ b$, then the ordered family of pairs $((a, 0),(0, b))$ is nice. If in addition we assume that $\operatorname{deg}(b) \geq 1$, then also the family $\left((a,-b),\left(S_{h} a,-b\right),\left(0, S_{h} b-b\right)\right)$ is nice for every $h \in \mathbb{N}$. This is a special case of a more general phenomenon that will be explained in Section 4.5]

4.2.2. The van der Corput operation. Given an ordered family of pairs of functions $(\mathcal{A}, \mathcal{B})$, a pair of functions $(a, b)$, and $h \in \mathbb{N}$, we define the following operation:

$$
\begin{aligned}
(a, b, h)-\operatorname{vdC}(\mathcal{A}, \mathcal{B}):=\left(\left(S_{h} a_{1}-a, S_{h} b_{1}-b\right)\right. & , \ldots,\left(S_{h} a_{m}-a, S_{h} b_{m}-b\right), \\
& \left.\left(a_{1}-a, b_{1}-b\right), \ldots,\left(a_{m}-a, b_{m}-b\right)\right)^{*},
\end{aligned}
$$

where ${ }^{*}$ is the operation that removes all pairs of bounded functions.

4.3. Strategy of proof of Proposition 4.2, Our proof strategy of Proposition 4.2 is to successively apply Lemma 3.7 in order to bound the $L^{2}(\mu)$ norm of the averages in question with the $L^{2}(\mu)$ norm of averages that are simpler to deal with. In order to carry out this reduction a key step is to show that given a nice family of pairs $(\mathcal{A}, \mathcal{B})$ with $\operatorname{deg}\left(a_{1}\right) \geq 1$, it is always possible to find $(\tilde{a}, \tilde{b}) \in(\mathcal{A}, \mathcal{B})$ such that for all large enough $h \in \mathbb{N}$ the operation $(\tilde{a}, \tilde{b}, h)$-vdC leads to a nice family of pairs that has smaller type. Eventually, this procedure leads to families of pairs with sub-linear growth (i.e. with degree 0), in which case Proposition 4.2 can be established directly in a relatively simple manner.

We explain how this reduction to the degree 0 case works in the next example:

Example. Our goal is to find $k \in \mathbb{N}$ such that if $\left\|f_{1}\right\|_{k, T_{1}}=0$, then the averages

$$
\frac{1}{N} \sum_{n=1}^{N} f_{1}\left(T_{1}^{\left[n^{1.5}\right]} x\right) \cdot f_{2}\left(T_{2}^{\left[n^{1.1}\right]} x\right)
$$

converge to 0 in $L^{2}(\mu)$ as $N \rightarrow \infty$. 
We define $\mathcal{A}=\left(t^{1.5}, 0\right), \mathcal{B}=\left(0, t^{1.1}\right)$, and introduce the following nice family of pairs of functions:

$$
(\mathcal{A}, \mathcal{B})=\left(\left(t^{1.5}, 0\right),\left(0, t^{1.1}\right)\right)
$$

This family is nice and has type $\left(\begin{array}{ll}1 & 0 \\ 1 & 0\end{array}\right)$. Applying the $\mathrm{vdC}$ operation with $(a, b)=$ $\left(0, t^{1.1}\right)$, we see that for $h \in \mathbb{N}$, the ordered family $(a, b, h)-\operatorname{vdC}(\mathcal{A}, \mathcal{B})$ is equal to

$$
\left(\left((t+h)^{1.5},-t^{1.1}\right),\left(0,(t+h)^{1.1}-t^{1.1}\right),\left(t^{1.5},-t^{1.1}\right)\right) .
$$

The important point is that for every $h \in \mathbb{N}$ this new family is also nice and has smaller type, namely $\left(\begin{array}{ll}1 & 0 \\ 0 & 1\end{array}\right)$. Loosely speaking, one expects to be able to show (using Lemma 3.7) that the averages (21) converge to 0 in $L^{2}(\mu)$ once one can show that for every $h \in \mathbb{N}$ the averages

$$
\frac{1}{N} \sum_{n=1}^{N} f_{1}\left(T_{1}^{\left[(n+h)^{1.5}\right]} T_{2}^{\left[-n^{1.1}\right]} x\right) \cdot \tilde{g}\left(T_{2}^{\left[(n+h)^{1.1}-n^{1.1}\right]} x\right) \cdot \tilde{h}\left(T_{1}^{\left[n^{1.5}\right]} T_{2}^{\left[-n^{1.1}\right]} x\right)
$$

converge to 0 in $L^{2}(\mu)$ for all $\tilde{g}, \tilde{h} \in L^{\infty}(\mu)$.

For $h \in \mathbb{N}$, applying the $\mathrm{vdC}$ operation one more time with $(a, b)=$ $\left(0,(t+h)^{1.1}-t^{1.1}\right)$ leads to a nice ordered family with 4 pairs and type $\left(\begin{array}{ll}1 & 0 \\ 0 & 0\end{array}\right)$. Lastly, for $h \in \mathbb{N}$, applying the vdC operation one more time with $(a, b)=\left(t^{1.5},-(t+h)^{1.1}\right)$, we are led to a nice ordered family with 7 pairs and type $\left(\begin{array}{ll}0 & 7 \\ 0 & 0\end{array}\right)$. In this case all functions involved have sub-linear growth, and the iterates of $T_{1}$ grow faster than any of the iterates of $T_{2}$. Taking advantage of this fact, we can show in a relatively simple way that the corresponding multiple ergodic averages converge to 0 in $L^{2}(\mu)$ if $\left\|f_{1}\right\|_{16, T_{1}}=0$.

4.4. Two technical lemmas. We establish two simple results that will be used repeatedly.

Lemma 4.8. Let $a:[c, \infty) \rightarrow \mathbb{R}$ be a Hardy field function with non-negative degree $d$ and let $b \in \mathcal{F}(a)$. Then either $b(t) \rightarrow 0$, or there exists $k \in\{0, \ldots, d\}$ such that $b \sim a / t^{k}$.

Proof. Without loss of generality we can assume that $a(t) \rightarrow \infty$. Suppose that

$$
b=\sum_{i=1}^{l} k_{i} \cdot S_{h_{i}} a .
$$

Since $\operatorname{deg}(a)=d$ we have by Lemma 3.2 that $a^{(d+1)}(t) \rightarrow 0$. Using this and Lagrange's remainder formula for the Taylor series of the function $a(t)$, we see that for $h \in \mathbb{N}$ we have

$$
S_{h} a=\sum_{i=0}^{d} a^{(i)} h^{i} / i !+e_{h},
$$

where $e_{h}:[c, \infty) \rightarrow \mathbb{R}$ is a function that satisfies $e_{h}(t) \rightarrow 0$. Combining the above identities we deduce that

$$
b=\sum_{i=0}^{d} c_{i} a^{(i)}+e
$$

for some constants $c_{i} \in \mathbb{R}$ and function $e:[c, \infty) \rightarrow \mathbb{R}$ that satisfies $e(t) \rightarrow 0$. If $c_{i}=0$ for $i=0, \ldots, d$, then $b(t) \rightarrow 0$. Otherwise, let $i_{0}$ be the smallest $i$ such that $c_{i} \neq 0$. Then $b \sim a^{\left(i_{0}\right)}$, and by Lemma 3.2 we have $a^{\left(i_{0}\right)} \sim a / t^{i_{0}}$. Taking $d=i_{0}$ completes the proof. 
Lemma 4.9. Let $a:[c, \infty) \rightarrow \mathbb{R}$ be a Hardy field function with polynomial growth rate and $a_{1}, a_{2} \in \mathcal{F}(a)$ be such that $a_{1} \succ t^{\varepsilon}$ for some $\varepsilon>0$ and $a_{2} \ll a_{1}$.

(i) If $a_{1} \not a_{2}$, then $S_{h} a_{1}-a_{2} \sim a_{1}$ for every non-zero $h \in \mathbb{R}$.

(ii) If $a_{1} \cong a_{2}$, then $S_{h} a_{1}-a_{2} \ll a_{1} / t$ for every $h \in \mathbb{R}$, and $S_{h} a_{1}-a_{2} \sim a_{1} / t$ for all but one $h \in \mathbb{R}$.

Remark. The assumption $a_{1}, a_{2} \in \mathcal{F}(a)$ is necessary. To see this, for $(i)$ take $a_{1}(t)=t^{1.5}+t^{1.1}, a_{2}(t)=t^{1.5}$, and for $(i i)$ take $a_{1}(t)=t^{1.5}+t^{0.9}, a_{2}(t)=t^{1.5}$.

Proof. We prove $(i)$. Suppose on the contrary that $S_{h} a_{1}-a_{2} \nsim a_{1}$ for some $h \in \mathbb{R}$. Since $S_{h} a_{1}-a_{2} \ll a_{1}$, we deduce that $S_{h} a_{1}-a_{2} \prec a_{1}$.

We claim that $S_{h} a_{1}-a_{2} \ll a_{1} / t$. Indeed, by Lemma 4.8 we have $a_{1} \sim a / t^{k}$ for some non-negative integer $k$. Since $S_{h} a_{1}-a_{2} \in \mathcal{F}(a)$, Lemma 4.8 gives that either $S_{h} a_{1}-a_{2} \prec 1$, or $S_{h} a_{1}-a_{2} \sim a / t^{k^{\prime}}$ for some non-negative integer $k^{\prime}$. If $S_{h} a_{1}-a_{2} \prec 1$, then the claim is proved because $\operatorname{deg}\left(a_{1}\right) \geq 1$. If $S_{h} a_{1}-a_{2} \sim a / t^{k^{\prime}}$, then since $S_{h} a_{1}-a_{2} \prec a_{1} \sim a / t^{k}$, we deduce that $k^{\prime}>k$, proving the claim.

Using the previous claim, Lemma [3.2, and expressing $a_{1}-a_{2}$ as $\left(a_{1}-S_{h} a_{1}\right)+$ $\left(S_{h} a_{1}-a_{2}\right)$, we deduce that $a_{1}-a_{2} \ll a_{1} / t$. This is a contradiction since by assumption $a_{1} \nRightarrow a_{2}$.

We prove (ii). Expressing $S_{h} a_{1}-a_{2}$ as $\left(S_{h} a_{1}-a_{1}\right)+\left(a_{1}-a_{2}\right)$ and using Lemma 3.2 and our assumption $a_{1} \cong a_{2}$, we see that for every $h \in \mathbb{R}$ we have $S_{h} a_{1}-a_{2} \prec a_{1}$. From this we deduce as in the proof of part $(i)$ that $S_{h} a_{1}-a_{2} \ll a_{1} / t$ for every $h \in \mathbb{R}$. It remains to show that if $S_{h_{0}} a_{1}-a_{2} \prec a_{1} / t$ for some $h_{0} \in \mathbb{R}$, then $S_{h} a_{1}-a_{2} \sim a_{1} / t$ for every $h \neq h_{0}$. To see this, we express $S_{h} a_{1}-a_{2}$ as $\left(S_{h} a_{1}-S_{h_{0}} a_{1}\right)+\left(S_{h_{0}} a_{1}-a_{2}\right)$, and use that by Lemma 3.2 we have $S_{h} a_{1}-a_{1} \sim a_{1} / t$ for every non-zero $h \in \mathbb{R}$. This completes the proof.

4.5. Reducing the type. The next lemma is a key ingredient of the proof of Proposition 4.2

Lemma 4.10. Let $(\mathcal{A}, \mathcal{B})$ be a nice family of pairs of functions, and suppose that $\operatorname{deg}\left(a_{1}\right) \geq 1$. Then there exist $\tilde{a} \in \mathcal{A} \cup\{0\}$ and $\tilde{b} \in \mathcal{B}$, such that for every large enough $h \in \mathbb{N}$, the family $(\tilde{a}, \tilde{b}, h)-\operatorname{vdC}(\mathcal{A}, \mathcal{B})$ is nice and has type strictly smaller than that of $(\mathcal{A}, \mathcal{B})$.

Proof. By assumption, there exists a Hardy field $\mathcal{H}$, functions $a, b \in \mathcal{G} \cap \mathcal{H}$, and $a_{1}, \ldots, a_{m} \in \mathcal{F}(a), b_{1}, \ldots, b_{m} \in \mathcal{F}(b)$, such that $\mathcal{A}=\left(a_{1}, \ldots, a_{m}\right), \mathcal{B}=\left(b_{1}, \ldots, b_{m}\right)$. Given a pair of functions $(\tilde{a}, \tilde{b}) \in(\mathcal{A}, \mathcal{B})$ and $h \in \mathbb{N}$, the family $(\tilde{a}, \tilde{b}, h)-\operatorname{vdC}(\mathcal{A}, \mathcal{B})$ is an ordered family of pairs of functions, all of them of the form

$$
\left(S_{h} a_{i}-\tilde{a}, S_{h} b_{i}-\tilde{b}\right) \text {, or }\left(a_{i}-\tilde{a}, b_{i}-\tilde{b}\right) .
$$

We choose $(\tilde{a}, \tilde{b})$ as follows: If the family $\mathcal{B}^{\prime}$, defined by (20), is non-empty, then we take $\tilde{a}=0$ and let $\tilde{b}$ be a function in $\mathcal{B}^{\prime}$ with minimal degree. Then the first row of the matrix type remains unchanged, and one easily checks using Lemma 4.9 in the positive degree case and Lemma 3.2 in the 0 degree case, that the second row of the matrix type gets "reduced", leading to a smaller matrix type for every $h \in \mathbb{N}$. Suppose now that the family $\mathcal{B}^{\prime}$ is empty, in which case all the functions in the family $\mathcal{A}$ are unbounded. If $\mathcal{A}$ consists of a single function $a_{1}$, then we choose $(\tilde{a}, \tilde{b}):=\left(a_{1}, b_{1}\right)$ and the result follows. Therefore, we can assume that $\mathcal{A}$ contains a function other than $a_{1}$. We consider two cases. If $a_{i} \cong a_{1}$ for $i=2, \ldots, m$, then we choose $(\tilde{a}, \tilde{b}):=\left(a_{1}, b_{1}\right)$. Otherwise, we choose $(\tilde{a}, \tilde{b}) \in(\mathcal{A}, \mathcal{B})$ such that $\tilde{a} \nsucceq a_{1}$ 
and $\tilde{a}$ is a function in $\mathcal{A}^{\prime}$ (see (19)), with minimal degree (such a choice exists since $a_{1}$ has the highest degree in $\left.\mathcal{A}\right)$.

In all cases, for every $h \in \mathbb{N}$, one checks using Lemmas 3.2 and 4.9 that the first row of the matrix type of $(\tilde{a}, \tilde{b}, h)-\operatorname{vdC}(\mathcal{A}, \mathcal{B})$ is "smaller" than that of $(\mathcal{A}, \mathcal{B})$, and as a consequence the new family has strictly smaller type.

It remains to verify that for every large enough $h \in \mathbb{N}$ the ordered family of pairs of functions $(\tilde{a}, \tilde{b}, h)-\operatorname{vdC}(\mathcal{A}, \mathcal{B})$ is nice. We remark that, by construction, the first pair of functions in this family is $\left(S_{h} a_{1}-\tilde{a}, S_{h} b_{1}-\tilde{b}\right)$.

Claim. Property (1) of Definition 4.7 holds for all large enough $h \in \mathbb{N}$.

To prove the first part of property (1) it suffices to show that for all large enough $h \in \mathbb{N}$,

$$
S_{h} a_{1}-S_{h} a_{i} \rightarrow \infty \text { for } i=2, \ldots, m
$$

and

$$
S_{h} a_{1}-a_{i} \rightarrow \infty \text { for }=1, \ldots, m .
$$

The first property follows immediately from our assumption $a_{1}-a_{i} \rightarrow \infty$ for $i=1, \ldots, m$, and the second property follows upon observing that for all large enough $h \in \mathbb{N}$ we have by Lemma 4.9 that $a_{1} / t \ll S_{h} a_{1}-a_{i}$ and our assumption $\operatorname{deg}\left(a_{1}\right) \geq 1$ which combined with the property $a_{1} \in \mathcal{G}$ gives that $t \prec a_{1}$.

To prove the second part of property (1) it suffices to show that for all large enough $h \in \mathbb{N}$,

$$
S_{h} a_{i}-\tilde{a} \ll S_{h} a_{1}-\tilde{a} \text {, for } i=1, \ldots, m
$$

and

$$
a_{i}-\tilde{a} \ll S_{h} a_{1}-\tilde{a}, \text { for } i=1, \ldots, m .
$$

We only prove the first property; the second can be proved in a similar fashion. We consider two cases. If $\tilde{a} \not a_{1}$, then by Lemma 4.9 for all but one $h \in \mathbb{N}$ we have $S_{h} a_{1}-\tilde{a} \sim a_{1}$, and the estimate follows by our assumption $a_{i} \ll a_{1}$ for $i=1, \ldots, m$. If $\tilde{a} \cong a_{1}$, then by construction $\tilde{a} \cong a_{i}$ for $i=1, \ldots, m$. Therefore, for all large enough $h \in \mathbb{N}$ we have by Lemma 4.9 that $S_{h} a_{i}-\tilde{a} \sim a_{1} / t$ for $i=1, \ldots, m$. The result follows.

Claim. Property (2) of Definition 4.7 holds for all large enough $h \in \mathbb{N}$.

It suffices to show that for all large enough $h \in \mathbb{N}$,

$$
S_{h} b_{i}-\tilde{b} \prec S_{h} a_{1}-\tilde{a} \text {, for } i=1, \ldots, m
$$

and

$$
b_{i}-\tilde{b} \prec S_{h} a_{1}-\tilde{a}, \text { for } i=1, \ldots, m .
$$

We only prove the first property; the second one can be proved in a similar fashion. We consider two cases.

If $\tilde{a} \not a_{1}$, then by Lemma 4.9 for all but one $h \in \mathbb{N}$ we have $S_{h} a_{1}-\tilde{a} \sim a_{1}$, and so the result follows since by assumption $b_{i} \prec a_{1}$ for $i=1, \ldots, m$.

If $\tilde{a} \cong a_{1}$, then by construction $(\tilde{a}, \tilde{b})=\left(a_{1}, b_{1}\right)$ and $a \cong a_{i}$ for $i=1, \ldots, m$. It therefore remains to show that for all large enough $h \in \mathbb{N}$ we have $S_{h} b_{i}-b_{1} \prec$ $S_{h} a_{1}-a_{1}$ for $i=1, \ldots, m$. To see this, we express $S_{h} b_{i}-b_{1}$ as $\left(S_{h} b_{i}-b_{i}\right)+\left(b_{i}-b_{1}\right)$. If $1 \prec b_{i}$, then $b_{i} \in \mathcal{G}$ (by Lemma 4.8) and Lemma 3.2 gives that for every $h \in \mathbb{N}$ we have $S_{h} b_{i}-b_{i} \ll b_{i} / t \prec a_{1} / t$. If $b_{i} \ll 1$, then since $t \prec a_{1}$ we still get $S_{h} b_{i}-b_{i} \prec a_{1} / t$. Furthermore, for $i=2, \ldots, m$, by assumption we have $b_{i}-b_{1} \prec a_{i}-a_{1}$ and by Lemma 4.9 we have $a_{i}-a_{1} \ll a_{1} / t$. Combining the above we get for every $h \in \mathbb{N}$ 
that $S_{h} b_{i}-b_{1} \prec a_{1} / t$ for $i=1, \ldots, m$. Since by Lemma 3.2 for every $h \in \mathbb{N}$ we have $S_{h} a_{1}-a_{1} \sim a_{1} / t$, the result follows.

Claim. Property (3) of Definition 4.7 holds for all large enough $h$.

Equivalently, we claim that for all large enough $h \in \mathbb{N}$,

$$
S_{h} b_{1}-S_{h} b_{i} \prec S_{h} a_{1}-S_{h} a_{i} \text {, for } i=2, \ldots, m,
$$

and

$$
S_{h} b_{1}-b_{i} \prec S_{h} a_{1}-a_{i}, \text { for } i=1, \ldots, m .
$$

The first property follows immediately from our hypothesis $b_{1}-b_{i} \prec a_{1}-a_{i}$ for $i=2, \ldots, m$. We verify the second property. If $a_{i} \not a_{1}$, then by Lemma 4.9 we have for all large enough $h \in \mathbb{N}$ that $S_{h} a_{1}-a_{i} \sim a_{1}$ for $i=2, \ldots, m$. The desired estimate now follows since by hypothesis $b_{i} \prec a_{1}$ for $i=1, \ldots, m$. Suppose now that $a_{i} \cong a_{1}$. Then Lemma 4.9 gives for all large enough $h \in \mathbb{N}$ that $S_{h} a_{1}-a_{i} \sim a_{1} / t$. So it remains to verify that for every large enough $h \in \mathbb{N}$ we have $S_{h} b_{1}-b_{i} \prec a_{1} / t$. To see this we express $S_{h} b_{1}-b_{i}$ as $\left(S_{h} b_{1}-b_{1}\right)+\left(b_{1}-b_{i}\right)$. Our assumptions and Lemma 3.2 give that $S_{h} b_{1}-b_{1} \sim b_{1} / t \prec a_{1} / t$ for all $h \in \mathbb{N}$. Furthermore, our assumptions and Lemma 4.9 give that $b_{1}-b_{i} \prec a_{1}-a_{i} \ll a_{1} / t$. Hence, for every $h \in \mathbb{N}$ we have $S_{h} b_{1}-b_{i} \prec a_{1} / t$, as desired. This completes the proof.

4.6. Some ergodic estimates. We gather here some simple ergodic estimates that will be used in the proof of Proposition 4.2 .

Using successive applications of Lemma 3.7 one can show the following (see for example Case 1 of Proposition 5.3 in [16]):

Lemma 4.11. Let $(X, \mathcal{X}, \mu, T)$ be a system, $f_{1}, \ldots, f_{m} \in L^{\infty}(\mu)$ be functions bounded by 1 , and $\alpha_{1}, \ldots, \alpha_{m}$ be non-zero integers such that $\alpha_{1} \neq \alpha_{i}$ for $i=$ $2, \ldots, m$. Then there exists $C:=C_{m, \alpha_{2}, \ldots, \alpha_{m}}$ such that

$$
\limsup _{N-M \rightarrow \infty} \sup _{\left\|f_{2}\right\|_{\infty}, \ldots,\left\|f_{m}\right\|_{\infty} \leq 1}\left\|\frac{1}{N-M} \sum_{n=M}^{N} \prod_{i=1}^{m} T^{\left[\alpha_{i} n\right]} f_{i}\right\|_{L^{2}(\mu)} \leq C\left\|f_{1}\right\|_{2 m, T} .
$$

The next two lemmas will help us handle bounded error terms that later on appear on the iterates of the transformations involved.

Lemma 4.12. Let $\left(X, \mathcal{X}, \mu, T_{1}, \ldots, T_{\ell}\right)$ be a system, $f_{1}, \ldots, f_{m} \in L^{\infty}(\mu)$ be functions, and for $i=1, \ldots, m, j=1, \ldots, \ell$, let $\left(a_{i, j}(n)\right)$ be sequences with integer values. Then for every $N \in \mathbb{N}$

$$
\begin{aligned}
\sup _{E \subset \mathbb{N}} & \left\|\frac{1}{N} \sum_{n=1}^{N} \prod_{i=1}^{m}\left(T_{1}^{a_{i, 1}(n)} \cdots T_{\ell}^{a_{i, \ell}(n)}\right) f_{i} \cdot \mathbf{1}_{E}(n)\right\|_{L^{2}(\mu)}^{2} \\
& \leq\left\|\frac{1}{N} \sum_{n=1}^{N} \prod_{i=1}^{m}\left(\tilde{T}_{1}^{a_{i, 1}(n)} \cdots \tilde{T}_{\ell}^{a_{i, \ell}(n)}\right) \tilde{f}_{i}\right\|_{L^{2}(\tilde{\mu})},
\end{aligned}
$$

where $\tilde{T}:=T \times T, \tilde{\mu}:=\mu \times \mu$, and $\tilde{f}:=f \otimes \bar{f}$.

Proof. Letting

$$
F_{n}:=\prod_{i=1}^{m}\left(T_{1}^{a_{i, 1}(n)} \cdots T_{\ell}^{a_{i, \ell}(n)}\right) f_{i}
$$


we see that the left hand side in (22) is bounded by

$$
\frac{1}{N^{2}} \sum_{1 \leq m, n \leq N}\left|\int F_{n} \cdot \bar{F}_{m} d \mu\right| \text {. }
$$

It follows that the square of the left hand side in (22) is bounded by

$$
\frac{1}{N^{2}} \sum_{1 \leq m, n \leq N}\left|\int F_{n} \cdot \bar{F}_{m} d \mu\right|^{2}=\frac{1}{N^{2}} \sum_{1 \leq m, n \leq N} \int G_{n} \cdot \bar{G}_{m} d \tilde{\mu}=\left\|\frac{1}{N} \sum_{n=1}^{N} G_{n}\right\|_{L^{2}(\tilde{\mu})}^{2},
$$

where

$$
G_{n}:=\prod_{i=1}^{m}\left(\tilde{T}_{1}^{a_{i, 1}(n)} \cdots \tilde{T}_{\ell}^{a_{i, \ell}(n)}\right) \tilde{f}_{i} .
$$

This completes the proof.

We deduce from this the following:

Lemma 4.13. Let $\left(X, \mathcal{X}, \mu, T_{1}, \ldots, T_{\ell}\right)$ be a system, $f_{1}, \ldots, f_{m} \in L^{\infty}(\mu)$ be functions, and for $i=1, \ldots, m, j=1, \ldots, \ell$, let $\left(a_{i, j}(n)\right)$ be sequences with integer values and $\left(e_{i, j}(n)\right)$ be sequences that take values in some finite set of integers $F$. Then for every $N \in \mathbb{N}$

$$
\begin{gathered}
\sup _{E \subset \mathbb{N}}\left\|\frac{1}{N} \sum_{n=1}^{N} \prod_{i=1}^{m}\left(T_{1}^{a_{i, 1}(n)+e_{i, 1}(n)} \cdots T_{\ell}^{a_{i, \ell}(n)+e_{i, \ell}(n)}\right) f_{i} \cdot \mathbf{1}_{E}(n)\right\|_{L^{2}(\mu)}^{2} \\
\leq|F|^{2 \ell m} \cdot \max _{c_{i, j} \in F}\left\|\frac{1}{N} \sum_{n=1}^{N} \prod_{i=1}^{m}\left(\tilde{T}_{1}^{a_{i, 1}(n)+c_{i, 1}} \cdots \tilde{T}_{\ell}^{a_{i, \ell}(n)+c_{i, \ell}}\right) \tilde{f}_{i}\right\|_{L^{2}(\tilde{\mu})},
\end{gathered}
$$

where $\tilde{T}:=T \times T, \tilde{\mu}:=\mu \times \mu$, and $\tilde{f}:=f \otimes \bar{f}$.

Proof. The $L^{2}(\mu)$ norm on left hand side is less than

$$
\sum_{j=1}^{t}\left\|\frac{1}{N} \sum_{n=1}^{N} \prod_{i=1}^{m}\left(T_{1}^{a_{i, 1}(n)+e_{i, 1}(n)} \cdots T_{\ell}^{a_{i, \ell}(n)+e_{i, \ell}(n)}\right) f_{i} \cdot \mathbf{1}_{E_{j}}(n)\right\|_{L^{2}(\mu)}
$$

where the sets $E_{1}, \ldots, E_{t}\left(t \leq|F|^{\ell m}\right)$ form a partition of $E$ into sets where the sequences $e_{i, j}$ are all constant. The desired estimate is now an immediate consequence of Lemma 4.12

4.7. Proof of Proposition 4.2, We start with an elementary lemma that will be used to prove seminorm estimates in the case where all the iterates have sub-linear growth.

Lemma 4.14. Let $a:[c, \infty) \rightarrow \mathbb{R}$ be a positive Hardy field function that satisfies the growth condition $\log t \prec a(t) \prec t$ and $(A(n))$ be a bounded sequence in a normed space such that $\lim _{N-M \rightarrow \infty}\left\|\frac{1}{N-M} \sum_{n=M}^{N} A(n)\right\|=0$. Then

$$
\lim _{N \rightarrow \infty}\left\|\frac{1}{N} \sum_{n=1}^{N} A([a(n)])\right\|=0 .
$$

Remark. When $t^{\varepsilon} \prec a(t) \prec 1$ for some $\varepsilon>0$, the conclusion holds under the weaker assumption $\lim _{N \rightarrow \infty}\left\|\frac{1}{N} \sum_{n=1}^{N} A(n)\right\|=0$. 
Proof. Letting $w(n)=\{k \in \mathbb{N}:[a(k)]=n\}$ and $W(N)=w(1)+\cdots+w(N)$, it suffices to show that $\lim _{N \rightarrow \infty}\left\|\frac{1}{W(N)} \sum_{n=1}^{N} w(n) \cdot A(n)\right\|=0$. Letting $b(t)=$ $a^{-1}(t)$, one checks that $w(n) /(b(n+1)-b(n)) \rightarrow 1$ and $W(n) / b(n) \rightarrow 1$. Our assumptions give that $\log (b(t)) \prec t \prec b(t)$. This implies that $b(t+1)-b(t) \rightarrow \infty$ and $(b(t+1)-b(t)) / b(t) \rightarrow 0$. Hence, $w(n) \rightarrow \infty$ and $w(n) / W(n) \rightarrow 0$. The needed convergence to 0 now follows from Theorem 3.6 in [5].

We are now in a position to prove Proposition 4.2. Given a Hardy field $\mathcal{H}$ and functions $a, b \in \mathcal{G} \cap \mathcal{H}$ our goal is to establish the following claim:

Claim. Let $a_{i} \in \mathcal{F}(a), b_{i} \in \mathcal{F}(b)$ for $i=1, \ldots, m$, and let $(\mathcal{A}, \mathcal{B})$ be a nice family of ordered pairs of functions where $\mathcal{A}:=\left(a_{1}, \ldots, a_{m}\right), \mathcal{B}:=\left(b_{1}, \ldots, b_{m}\right)$. Let $W$ be the matrix type of this family. Then there exists $k=k(W, m) \in \mathbb{N}$ such that: If $\left\|f_{1}\right\|_{k, T_{1}}=0$, then the averages

$$
\frac{1}{N} \sum_{n=1}^{N} \prod_{i=1}^{m}\left(T_{1}^{\left[a_{i}(n)\right]} T_{2}^{\left[b_{i}(n)\right]}\right) f_{i}
$$

converge to 0 in $L^{2}(\mu)$.

Note that the conclusion of Proposition 4.2 is somewhat stronger in two respects: (i) The integer $k$ depends only on the degree of the family. This strengthening easily follows from the above mentioned claim after noticing that there is only a finite number of possible matrix types for families that have fixed degree and numbers of pairs of functions. (ii) The conclusion involves a supremum over all subsets of $\mathbb{N}$. This strengthening follows by combining the above mentioned statement with Lemma 4.12 and the fact that $\|f\|_{k+1, T}=0$ implies that $\|f \otimes \bar{f}\|_{k, T \times T}=0$ (this follows from (15)).

We proceed to prove the claim by induction on the type of the nice family $(\mathcal{A}, \mathcal{B})$.

Base case. Suppose that $\operatorname{deg}\left(a_{1}\right)=0$, in which case, for $i=1, \ldots, m$ the functions $a_{i}$ and $b_{i}$ have sub-linear growth. We are going to show that if $\left\|f_{1}\right\|_{2 m+1, T_{1}}=0$, then the averages (23) converge to 0 in $L^{2}(\mu)$.

Our assumption implies that for $i=2, \ldots, m$ one has

$$
a_{i}(t)=\alpha_{i} a_{1}(t)+c_{i}(t)
$$

for some $\alpha_{i} \in \mathbb{R}$ and functions $c_{i}$ that satisfy $c_{i} \prec a_{1}$. It is important to note that $\alpha_{i} \neq 1$ for $i=2, \ldots, m$. Otherwise $a_{1}-a_{i} \prec a_{1}$, and since $a_{1}-a_{i} \in \mathcal{F}(a)$ and $\operatorname{deg}\left(a_{1}\right)=0$, we deduce by Lemma 4.8 that $a_{1}-a_{i} \rightarrow 0$, contradicting our assumption that the family $(\mathcal{A}, \mathcal{B})$ is nice. Let

$$
\tilde{b}_{i}:=b_{i} \circ a_{1}^{-1}, \quad \tilde{c}_{i}:=c_{i} \circ a_{1}^{-1} .
$$

(We caution the reader that these functions are not necessarily Hardy field functions.) Since $b_{i} \prec a_{1}$ and $c_{i} \prec a_{1}$ we have $\tilde{b}_{i} \prec 1$ and $\tilde{c}_{i} \prec 1$. Furthermore, one sees that

$$
\left[a_{i}(n)\right]=\left[\alpha_{i}\left[a_{1}(n)\right]\right]+\left[\tilde{c}_{i}\left(\left[a_{1}(n)\right]\right)\right]+e_{i}(n), \quad\left[b_{i}(n)\right]=\left[\tilde{b}_{i}\left(\left[a_{1}(n)\right]\right)\right]+e_{i}^{\prime}(n),
$$


where the sequences $\left(e_{i}(n)\right),\left(e_{i}^{\prime}(n)\right)$ take finitely many integer values. Therefore, it suffices to show that the averages in $n$ of

$$
\left(T_{1}^{\left[a_{1}(n)\right]} T_{2}^{\left[\tilde{b}_{1}\left(\left[a_{1}(n)\right]\right)\right]+e_{1}^{\prime}(n)}\right) f_{1} \cdot \prod_{i=2}^{m}\left(T_{1}^{\left[\alpha_{i}\left[a_{1}(n)\right]\right]+\left[\tilde{c}_{i}\left(\left[a_{1}(n)\right]\right)\right]+e_{i}(n)} T_{2}^{\left[\tilde{b}_{i}\left(\left[a_{1}(n)\right]\right)\right]+e_{i}^{\prime}(n)}\right) f_{i}
$$

converge to 0 in $L^{2}(\mu)$.

By Lemma 4.13 it suffices to show that the averages in $n$ of

$$
\left(\tilde{T}_{1}^{\left[a_{1}(n)\right]} \tilde{T}_{2}^{\left[\tilde{b}_{1}\left(\left[a_{1}(n)\right]\right)\right]}\right) \tilde{f}_{1} \cdot \prod_{i=2}^{m}\left(\tilde{T}_{1}^{\left[\alpha_{i}\left[a_{1}(n)\right]\right]+\left[\tilde{c}_{i}\left(\left[a_{1}(n)\right]\right)\right]} \tilde{T}_{2}^{\left[\tilde{b}_{i}\left(\left[a_{1}(n)\right]\right)\right]}\right) \tilde{f}_{i}
$$

converge to 0 in $L^{2}(\tilde{\mu})$ for all $\tilde{f}_{i} \in L^{\infty}(\tilde{\mu}), i=2, \ldots, m$, where $\tilde{T}:=T \times T, \tilde{\mu}:=\mu \times \mu$, and $\tilde{f}:=f \otimes \bar{f}$. Using Lemma 4.14 we can further reduce matters to show that for every sequence $\left(I_{N}\right)$ of intervals of integers with lengths increasing to infinity, the averages

$$
\frac{1}{\left|I_{N}\right|} \sum_{n \in I_{N}}\left(\tilde{T}_{1}^{n} \tilde{T}_{2}^{\left[\tilde{b}_{1}(n)\right]}\right) \tilde{f}_{1} \cdot \prod_{i=2}^{m}\left(\tilde{T}_{1}^{\left[\alpha_{i} n\right]+\left[\tilde{c}_{i}(n)\right]} \tilde{T}_{2}^{\left[\tilde{b}_{i}(n)\right]}\right) \tilde{f}_{i}
$$

converge to 0 in $L^{2}(\tilde{\mu})$ as $N \rightarrow \infty$.

Using our assumptions, one easily sees that the functions $\tilde{c}_{i}(t+1)-\tilde{c}_{i}(t)$ and $\tilde{b}_{i}(t+1)-\tilde{b}_{i}(t)$ converge to 0 and eventually have constant sign. Because of this, it is possible to decompose each interval $I_{N}$ (except a finite set with fixed cardinality) into sub-intervals with lengths increasing to infinity, and such that for every $N \in \mathbb{N}$ the sequences $\left(\left[\tilde{c}_{2}(n)\right]\right), \ldots,\left(\left[\tilde{c}_{m}(n)\right]\right)$ and $\left(\left[\tilde{b}_{1}(n)\right]\right), \ldots,\left(\left[\tilde{b}_{m}(n)\right]\right)$ are constant on each interval. Thus, without loss of generality we can assume that all these sequences are constant in each interval $I_{N}$. Then the desired fact would follow if we prove that the averages

$$
\frac{1}{\left|I_{N}\right|} \sum_{n \in I_{N}} \tilde{T}_{1}^{n} \tilde{f}_{1} \cdot \prod_{i=2}^{m}\left(\tilde{T}_{1}^{\left[\alpha_{i} n\right]+c_{i, N}} \tilde{T}_{2}^{d_{i, N}}\right) \tilde{f}_{i}
$$

converge to 0 in $L^{2}(\tilde{\mu})$ as $N \rightarrow \infty$, for every choice of integers $c_{i, N}, d_{i, N}$. This follows from Lemma 4.11 and the fact that $\left\|f_{i}\right\|_{2 m+1, T_{i}}=0$ implies that $\left\|\tilde{f}_{i}\right\|_{2 m, \tilde{T}_{i}}=0$.

Inductive step. Now let $(\mathcal{A}, \mathcal{B})$ be a nice family of $m$ ordered pairs of functions, of matrix type $W$, and such that $\operatorname{deg}\left(a_{1}\right) \geq 1$. Suppose that the statement we want to prove holds for every nice family of $2 \mathrm{~m}$ ordered pairs of functions with matrix type $W^{\prime}$ strictly less than $W$ (there is a finite number of such families), and let $k\left(W^{\prime}, 2 m\right)$ be the integer for which the conclusion of the corresponding statement holds. We let $k(W, m):=\max _{W^{\prime}<W}\left(k\left(W^{\prime}, 2 m\right)\right)+1$. Our goal is to show that $k(W, m)$ works for the family $(\mathcal{A}, \mathcal{B})$. Since in the base case we covered all nice families with degree 0 , this is going to complete the induction.

So assuming that $\left\|f_{1}\right\|_{k(W, m), T_{1}}=0$, we want to show that the averages (23) converge to 0 in $L^{2}(\mu)$. By Lemma 3.7 it suffices to show that for large enough $h \in \mathbb{N}$ the averages in $n$ of

$$
\int \prod_{i=1}^{m}\left(T_{1}^{\left[a_{i}(n+h)\right]} T_{2}^{\left[b_{i}(n+h)\right]}\right) f_{i} \cdot\left(T_{1}^{\left[a_{i}(n)\right]} T_{2}^{\left[b_{i}(n)\right]}\right) \bar{f}_{i} d \mu
$$


converge to 0 . We compose with $T_{1}^{-[\tilde{a}(n)]} T_{2}^{-[\tilde{b}(n)]}$, where $(\tilde{a}, \tilde{b}) \in(\mathcal{A}, \mathcal{B})$ is chosen as in Lemma 4.10, and use the Cauchy-Schwarz inequality. This reduces matters to show that for every large enough $h \in \mathbb{N}$ the averages in $n$ of

$$
\begin{gathered}
\prod_{i=1}^{m}\left(T_{1}^{\left[a_{i}(n+h)-\tilde{a}(n)\right]+e_{1, i}(n)} T_{2}^{\left[b_{i}(n+h)-\tilde{b}(n)\right]+e_{2, i}(n)}\right) f_{i} \\
\cdot\left(T_{1}^{\left[a_{i}(n)-\tilde{a}(n)\right]+e_{3, i}(n)} T_{2}^{\left[b_{i}(n)-\tilde{b}(n)\right]+e_{4, i}(n)}\right) \bar{f}_{i}
\end{gathered}
$$

converge to 0 in $L^{2}(\mu)$, where $e_{i, j}$ are sequences that take values in the set $\{0,1\}$. By Lemma 4.13 it suffices to show that the averages in $n$ of

(24)

$$
\prod_{i=1}^{m}\left(\tilde{T}_{1}^{\left[a_{i}(n+h)-\tilde{a}(n)\right]+c_{1, i}} \tilde{T}_{2}^{\left[b_{i}(n+h)-\tilde{b}(n)\right]+c_{2, i}}\right) \tilde{f}_{i} \cdot\left(\tilde{T}_{1}^{\left[a_{i}(n)-\tilde{a}(n)\right]+c_{3, i}} \tilde{T}_{2}^{\left[b_{i}(n)-\tilde{b}(n)\right]+c_{4, i}}\right) \overline{\tilde{f}}_{i}
$$

converge to 0 in $L^{2}(\tilde{\mu})$, where, $c_{i, j}$ are constants with values either 0 or $1, c_{1,1}=$ $c_{2,1}=0$, and $\tilde{T}:=T \times T, \tilde{\mu}:=\mu \times \mu, \tilde{f}:=f \otimes \bar{f}$. We remove the functions that happen to be composed with eventually constant iterates of $T_{1}$ and $T_{2}$ (this will happen when the functions involved are bounded), since they do not affect convergence to 0. This corresponds to the operation * defined in Section 4.2.2, and the resulting multiple ergodic averages are associated with the families of functions $(\tilde{a}, \tilde{b}, h)-\operatorname{vdC}(\mathcal{A}, \mathcal{B})$. Our final goal is to show that these averages convergence to 0 in $L^{2}(\mu)$ for every large enough $h \in \mathbb{N}$.

By Lemma 4.10, for every large enough $h \in \mathbb{N}$, the family $(\tilde{a}, \tilde{b}, h)-\operatorname{vdC}(\mathcal{A}, \mathcal{B})$ is nice, has type $W^{\prime}$ strictly smaller than $W$, and its first pair is

$$
\left(\left[a_{1}(n+h)-\tilde{a}(n)\right],\left[b_{1}(n+h)-\tilde{b}(n)\right]\right) .
$$

Notice also that in (24) the iterate $T_{1}^{\left[a_{1}(n+h)-\tilde{a}(n)\right]} T_{2}^{\left[b_{1}(n+h)-\tilde{b}(n)\right]}$ is applied to the function $\tilde{f}_{1}$. Since $\left\|f_{1}\right\|_{k(W, m), T_{1}}=0$ implies that $\left\|\tilde{f}_{1}\right\|_{k\left(W^{\prime}, 2 m\right), \tilde{T}_{1}}=0$, the induction hypothesis applies and proves convergence to 0 in $L^{2}(\mu)$. This completes the proof of Proposition 4.2 .

\section{Seminorm estimates for the highest Degree iterate: THE GENERAL CASE}

The next proposition is the generalization of Proposition 4.2 to the case of an arbitrary number of transformations. To avoid unnecessary repetition, we define the concepts needed in the proof of Proposition 5.1, and then only summarize its proof providing details only when non-trivial modifications of the arguments used in the previous section are needed.

Proposition 5.1. Let $\left(X, \mathcal{X}, \mu, T_{1}, \ldots, T_{\ell}\right)$ be a system, and $f_{1}, \ldots, f_{m} \in L^{\infty}(\mu)$. Suppose that $\left(\mathcal{A}_{1}, \ldots, \mathcal{A}_{\ell}\right)$ is a nice ordered family of $\ell$-tuples of functions with degree $d$ (all notions are defined below). Then there exists $k=k(d, \ell, m) \in \mathbb{N}$ such that: If $\left\|f_{1}\right\|_{k, T_{1}}=0$, then

$$
\lim _{N \rightarrow \infty} \sup _{E \subset \mathbb{N}}\left\|\frac{1}{N} \sum_{n=1}^{N} \prod_{i=1}^{m}\left(T_{1}^{\left[a_{1, i}(n)\right]} \cdots T_{\ell}^{\left[a_{\ell, i}(n)\right]}\right) f_{i} \cdot \mathbf{1}_{E}(n)\right\|_{L^{2}(\mu)}=0 .
$$


Applying this result to the nice family $\left(\mathcal{A}_{1}, \ldots, \mathcal{A}_{\ell}\right)$ where $\mathcal{A}_{1}:=\left(a_{1}, 0, \ldots, 0\right)$, $\mathcal{A}_{2}:=\left(0, a_{2}, \ldots, 0\right), \ldots, \mathcal{A}_{\ell}:=\left(0, \ldots, 0, a_{\ell}\right)$, we get:

Proposition 5.2. Let $\left(X, \mathcal{X}, \mu, T_{1}, \ldots, T_{\ell}\right)$ be a system, and $f_{1}, \ldots, f_{\ell} \in L^{\infty}(\mu)$ be functions. Let $\mathcal{H}$ be a Hardy field and $a_{1}, \ldots, a_{\ell} \in \mathcal{G} \cap \mathcal{H}$ be functions with different growth and highest degree $d:=\operatorname{deg}\left(a_{1}\right)$. Then there exists $k=k(d, \ell)$ such that: If $\left\|f_{1}\right\|_{k, T_{1}}=0$, then the averages

$$
\frac{1}{N} \sum_{n=1}^{N} \prod_{i=1}^{\ell} T_{i}^{\left[a_{i}(n)\right]} f_{i}
$$

converge to 0 in $L^{2}(\mu)$.

\subsection{Families of $\ell$-tuples and their types.}

5.1.1. Families of $\ell$-tuples of functions. Let $\ell, m \in \mathbb{N}$. Given $\ell$ ordered families of functions

$$
\mathcal{A}_{1}:=\left(a_{1,1}, \ldots, a_{1, m}\right), \ldots, \mathcal{A}_{\ell}:=\left(a_{\ell, 1}, \ldots, a_{\ell, m}\right),
$$

we define an ordered family of $\ell$-tuples of functions as follows:

$$
\left(\mathcal{A}_{1}, \ldots, \mathcal{A}_{\ell}\right):=\left(\left(a_{1,1}, \ldots, a_{\ell, 1}\right), \ldots,\left(a_{1, m}, \ldots, a_{\ell, m}\right)\right) .
$$

The maximum of the degrees of the functions in the families $\mathcal{A}_{1}, \ldots, \mathcal{A}_{\ell}$ is called the degree of the family $\left(\mathcal{A}_{1}, \ldots, \mathcal{A}_{\ell}\right)$.

For convenience of exposition, if the $\ell$-tuples of bounded functions appear in $\left(\mathcal{A}_{1}, \ldots, \mathcal{A}_{\ell}\right)$ we remove them, and henceforth we assume:

All families $\left(\mathcal{A}_{1}, \ldots, \mathcal{A}_{\ell}\right)$ that we consider do not contain $\ell$-tuples of bounded functions.

5.1.2. Definition of type. We fix $d \geq 0$ and restrict ourselves to families of degree between 0 and $d$. We define

$$
\mathcal{A}_{1}^{\prime}:=\left\{a_{1, j} \in \mathcal{A}_{1}: a_{1, j} \text { is not bounded }\right\},
$$

and for $i=2, \ldots, \ell$,

$$
\mathcal{A}_{i}^{\prime}:=\left\{a_{i, j} \in \mathcal{A}_{i}: a_{i, j} \text { is not bounded and } a_{i^{\prime}, j} \text { is bounded for } i^{\prime}<i\right\} .
$$

For $i=1, \ldots, \ell$ and $j=0,1, \ldots, d$, we let $w_{i, j}$ be the number of distinct nonequivalent classes of functions of degree $j$ in the family $\mathcal{A}_{i}^{\prime}$. We define the (matrix) type of the family $\left(\mathcal{A}_{1}, \ldots, \mathcal{A}_{\ell}\right)$ to be the matrix

$$
\left(\begin{array}{ccc}
w_{1, d} & \ldots & w_{1,0} \\
w_{2, d} & \ldots & w_{2,0} \\
\vdots & \ldots & \vdots \\
w_{\ell, d} & \ldots & w_{\ell, 0}
\end{array}\right) .
$$

As in Section 4.1.3, we order these types lexicographically. The following extension of Lemma 4.5 holds:

Lemma 5.3. Every decreasing sequence of types of families of $\ell$-tuples is stationary. 


\subsection{Nice families and the van der Corput operation.}

5.2.1. Nice families. Henceforth, we are going to work with families of $\ell$-tuples of functions that satisfy the following properties:

Definition 5.4. Let $\mathcal{H}$ be a Hardy field, let $a_{1}, \ldots, a_{\ell} \in \mathcal{G} \cap \mathcal{H}$ be functions, let $a_{i, j} \in \mathcal{F}\left(a_{i}\right)$ for $i=1, \ldots, \ell, j=1, \ldots, m$, and let $\mathcal{A}_{1}:=\left(a_{1,1}, \ldots, a_{1, m}\right), \ldots, \mathcal{A}_{\ell}:=$ $\left(a_{\ell, 1}, \ldots, a_{\ell, m}\right)$. We call the ordered family $\left(\mathcal{A}_{1}, \ldots, \mathcal{A}_{\ell}\right)$ of $\ell$-tuples of functions nice if

(1) $a_{1,1}-a_{1, j} \succ 1$ and $a_{1, j} \ll a_{1,1}$ for $j=2, \ldots, m$;

(2) $a_{i, j} \prec a_{1,1}$ for $i=2, \ldots, \ell, j=1, \ldots, m$;

(3) $a_{i, 1}-a_{i, j} \prec a_{1,1}-a_{1, j}$ for $i=2, \ldots, \ell, j=2, \ldots, m$.

5.2.2. The van der Corput operation. Given a family $\mathcal{A}:=\left(a_{1}, \ldots, a_{m}\right)$, a function $a:[c, \infty) \rightarrow \mathbb{R}$, and $h \in \mathbb{N}$, we define

$$
S_{h} \mathcal{A}:=\left(S_{h} a_{1}, \ldots, S_{h} a_{m}\right) \text { and } \mathcal{A}-a:=\left(a_{1}-a, \ldots, a_{m}-a\right) .
$$

Given a family of $\ell$-tuples of functions $\left(\mathcal{A}_{1}, \ldots, \mathcal{A}_{\ell}\right)$, an $\ell$-tuple $\left(\tilde{a}_{1}, \ldots, \tilde{a}_{\ell}\right) \in$ $\left(\mathcal{A}_{1}, \ldots \mathcal{A}_{\ell}\right)$, and $h \in \mathbb{N}$, we define the following operation:

$$
\left(\tilde{a}_{1}, \ldots, \tilde{a}_{\ell}, h\right)-\operatorname{vdC}\left(\mathcal{A}_{1}, \ldots, \mathcal{A}_{\ell}\right):=\left(\tilde{\mathcal{A}}_{1, h}, \ldots, \tilde{\mathcal{A}}_{\ell, h}\right)^{*},
$$

where

$$
\tilde{\mathcal{A}}_{i, h}:=\left(S_{h} \mathcal{A}_{i}-\tilde{a}_{i}, \mathcal{A}_{i}-\tilde{a}_{i}\right)
$$

for $i=1, \ldots, \ell$, and ${ }^{*}$ is the operation that removes all $\ell$-tuples that consist of bounded functions from a given family of $\ell$-tuples of functions.

5.3. Reducing the type. The next lemma enables us to reduce the type of a nice family of $\ell$-tuples that has positive degree:

Lemma 5.5. Let $\left(\mathcal{A}_{1}, \ldots, \mathcal{A}_{\ell}\right)$ be a nice family of $\ell$-tuples of functions with $\operatorname{deg}\left(a_{1,1}\right)$ $\geq 1$. Then there exists $\left(\tilde{a}_{1}, \ldots, \tilde{a}_{\ell}\right) \in\left(\mathcal{A}_{1} \cup\{0\}, \ldots, \mathcal{A}_{\ell} \cup\{0\}\right)$ such that for every large enough $h \in \mathbb{N}$ the family $\left(\tilde{a}_{1}, \ldots, \tilde{a}_{\ell}, h\right)-\operatorname{vdC}\left(\mathcal{A}_{1}, \ldots, \mathcal{A}_{\ell}\right)$ is nice and has strictly smaller type than $\left(\mathcal{A}_{1}, \ldots, \mathcal{A}_{\ell}\right)$.

Proof. Let $\mathcal{A}_{i}:=\left(a_{i, 1}, \ldots, a_{i, m}\right)$ for $i=1, \ldots, \ell$. Let $i \in\{1, \ldots, \ell\}$ be the largest integer such that the family $\mathcal{A}_{i}^{\prime}$ is non-empty. We choose $\left(\tilde{a}_{1}, \ldots, \tilde{a}_{\ell}\right)$ as follows: If $i \neq 1$ (in which case $\mathcal{A}_{\ell}^{\prime}, \mathcal{A}_{\ell-1}^{\prime}, \ldots, \mathcal{A}_{i+1}^{\prime}$ are empty, and $\mathcal{A}_{i}^{\prime}$ is non-empty), then we take $\tilde{a}_{1}=\cdots=\tilde{a}_{i-1}=0$ and let $\tilde{a}_{i}$ be a function of minimal degree in $\mathcal{A}_{i}^{\prime}$. Then for every $h \in \mathbb{N}$, one checks using Lemmas 3.2 and 4.9 that the first $i-1$ rows of the matrix type remain unchanged, and the $i$-th row will get "reduced", leading to a smaller matrix type.

If $i=1$, then the families $\mathcal{A}_{\ell}^{\prime}, \mathcal{A}_{\ell-1}^{\prime}, \ldots, \mathcal{A}_{2}^{\prime}$ are all empty. If $\mathcal{A}_{1}$ consists of a single function, namely $a_{1,1}$, then we choose $\left(\tilde{a}_{1}, \ldots, \tilde{a}_{\ell}\right):=\left(a_{1,1}, \ldots, a_{\ell, 1}\right)$ and the result follows using Lemma 3.2. Therefore, we can assume that $\mathcal{A}_{1}$ contains some function other than $a_{1,1}$. We consider two cases. If $a \cong a_{1,1}$ for all $a \in \mathcal{A}_{1}$, then we choose $\left(\tilde{a}_{1}, \ldots, \tilde{a}_{\ell}\right):=\left(a_{1,1}, \ldots, a_{\ell, 1}\right)$. Otherwise, we choose $\left(\tilde{a}_{1}, \ldots, \tilde{a}_{\ell}\right) \in\left(\mathcal{A}_{1}, \ldots, \mathcal{A}_{\ell}\right)$ 
with $\tilde{a}_{1} ¥ a_{1,1}$, and such that $\tilde{a}_{1}$ is a function in $\mathcal{A}_{1}^{\prime}$ with minimal degree (such a choice exists since $a_{1,1}$ has the highest degree in $\mathcal{A}_{1}$ ). In all cases, for every $h \in \mathbb{N}$, one checks using Lemmas 3.2 and 4.9 that the first row of the matrix type of $\left(\tilde{a}_{1}, \ldots, \tilde{a}_{\ell}, h\right)-\operatorname{vdC}\left(\mathcal{A}_{1}, \ldots, \mathcal{A}_{\ell}\right)$ is "smaller" than that of $\left(\mathcal{A}_{1}, \ldots, \mathcal{A}_{\ell}\right)$.

It remains to verify that the family $\left(\tilde{a}_{1}, \ldots, \tilde{a}_{\ell}, h\right)-\operatorname{vdC}\left(\mathcal{A}_{1}, \ldots, \mathcal{A}_{\ell}\right)$ is nice for large enough $h \in \mathbb{N}$. This argument is very similar to the one used in Lemma 4.10 . and so we omit it.

5.4. Proof of Proposition 5.1. Proposition 5.1 is proved by an induction on the type of nice families of $\ell$-tuples of functions. The base case covers all families with degree 0 and is proved in a way completely analogous to the case $\ell=2$ that was treated in the previous section. The inductive step is also completely analogous to the case $\ell=2$ and is omitted.

\section{Correlation estimates}

In order to motivate the estimates that are proved in this section we recap part of our plan for studying the limiting behavior of the averages

$$
\frac{1}{N} \sum_{n=1}^{N} T_{1}^{\left[a_{1}(n)\right]} f_{1} \cdot T_{2}^{\left[a_{2}(n)\right]} f_{2}
$$

when $a_{2} \prec a_{1}$. We showed in Proposition 4.1 that there exists $d \in \mathbb{N}$ such that if $\left\|f_{1}\right\|_{d, T_{1}}=0$, then the averages (25) converge to 0 in $L^{2}(\mu)$. Our goal is to prove a similar result for the function $f_{2}$. Using the decomposition result of Proposition 3.4 we can reduce matters to show that there exists $d \in \mathbb{N}$ such that if $\left\|f_{2}\right\|_{d, T_{2}}=0$, then

$$
\frac{1}{N} \sum_{n=1}^{N} \mathcal{D}_{x}\left(\left[a_{1}(n)\right]\right) \cdot f_{2}\left(T_{2}^{\left[a_{2}(n)\right]} x\right) \rightarrow^{L^{2}(\mu)} 0,
$$

where $\left(\mathcal{D}_{x}(n)\right)$ is a uniformly bounded sequence of measurable functions such that for almost every $x \in X$ the sequence $\left(\mathcal{D}_{n}(x)\right)$ is a dual sequence of level at most $d$. This motivates us to seek estimates that connect averages of the form

$$
\frac{1}{N} \sum_{n=1}^{N} \mathcal{D}([a(n)]) \cdot A(n),
$$

where $(\mathcal{D}(n))$ is a dual sequence, and averages involving only a product of translates of the sequence $(A(n))$. We produce such estimates in this section.

\subsection{Correlation estimates for sequences.}

Proposition 6.1. Let $\mathcal{H}$ be a Hardy field and $b_{1}, \ldots, b_{l} \in \mathcal{H}$ be functions with maximum degree $d$. Let $(X, \mathcal{X}, \mu)$ be a probability space, $(A(n)),\left(\mathcal{D}_{1}(n)\right), \ldots,\left(\mathcal{D}_{l}(n)\right)$ be uniformly bounded sequences of $L^{\infty}(\mu)$ functions, such that for almost every $x \in X$, for $i=1, \ldots, l$, the sequences $\left(\mathcal{D}_{i, x}(n)\right)$ are dual sequences of level at most $r \in \mathbb{N}$. Then there exists $s_{0}:=s_{0}(d, l, r) \in \mathbb{N}$ and $C:=C(d, l, r) \in \mathbb{R}$ such that for some 
$s \leq s_{0}$ we have

$$
\begin{aligned}
\limsup _{N \rightarrow \infty} & \left\|\frac{1}{N} \sum_{n=1}^{N}\left(A_{x}(n) \cdot \prod_{i=1}^{l} \mathcal{D}_{i, x}\left(\left[b_{i}(n)\right]\right)\right)\right\|_{L^{2}(\mu)}^{2^{s}} \\
\leq & C \cdot \limsup _{H_{s} \rightarrow \infty} \frac{1}{H_{s}} \sum_{h_{s}=1}^{H_{s}} \cdots \limsup _{H_{1} \rightarrow \infty} \frac{1}{H_{1}} \\
& \cdot \sum_{h_{1}=1}^{H_{1}} \limsup _{N \rightarrow \infty} \sup _{E \subset \mathbb{N}}\left\|\frac{1}{N} \sum_{n=1}^{N} \prod_{\boldsymbol{\epsilon} \in\{0,1\}^{s}} \mathcal{C}^{|\boldsymbol{\epsilon}|} A_{x}(n+\boldsymbol{\epsilon} \cdot \mathbf{h}) \cdot \mathbf{1}_{E}(n)\right\|_{L^{2}(\mu)},
\end{aligned}
$$

where $\mathbf{h}:=\left(h_{1}, \ldots, h_{s}\right)$.

Remark. Notice that we do not have to assume that $b_{1}, \ldots, b_{l} \in \mathcal{G}$. When $\ell=1$ and $b_{1}(t)=t$ the result was proved in 27 .

Proof. To begin with, using identity (16), we see that there exist $k, \ell \in \mathbb{N}$ (in fact, $k:=l r$ and $\left.\ell:=l\left(2^{r}-1\right)\right)$, vector valued sequences of functions $\mathbf{b}_{1}, \ldots, \mathbf{b}_{\ell}:[c, \infty) \rightarrow$ $\mathbb{R}^{k}$, with coordinates functions $b_{i, j}$ taken from the set $\left\{0, b_{1}, \ldots, b_{l}\right\}$, and sequences $\mathbf{d}_{1}, \ldots, \mathbf{d}_{\ell}: \mathbb{N}^{k} \rightarrow L^{\infty}(\mu)$, such that

$$
\prod_{i=1}^{l} \mathcal{D}_{i, x}\left(\left[b_{i}(n)\right]\right)=\lim _{M \rightarrow \infty} \frac{1}{M^{k}} \sum_{\mathbf{m} \in[1, M]^{k}} \prod_{i=1}^{\ell} \mathbf{d}_{i, x}\left(\mathbf{m}+\left[\mathbf{b}_{i}(n)\right]\right),
$$

where $\left[\mathbf{b}_{i}\right]:=\left(\left[b_{i, 1}\right], \ldots,\left[b_{i, k}\right]\right)$ and $\mathbf{m}:=\left(m_{1}, \ldots, m_{k}\right)$. Furthermore, all functions $b_{i, j}$ and $\mathbf{d}_{i}$ are bounded by 1 . It therefore suffices to prove the following claim:

Claim. Let $k, \ell \in \mathbb{N}, \mathcal{H}$ be a Hardy field, and for $i=1, \ldots, \ell$ let $\mathbf{b}_{i}=\left(b_{i, 1}, \ldots, b_{i, k}\right)$, where $b_{i, j} \in \mathcal{H}$ are functions with maximum degree $d$. Furthermore, let $(A(n))$, $\left(\mathbf{d}_{1}(\mathbf{m})\right), \ldots,\left(\mathbf{d}_{\ell}(\mathbf{m})\right), \mathbf{m} \in \mathbb{N}^{k}$, be sequences of $L^{\infty}(\mu)$ functions, all bounded by 1. Then there exists $s_{0}:=s_{0}(d, k, \ell) \in \mathbb{N}$ such that for some $s \leq s_{0}$ the expression

(26) $\quad \limsup _{N \rightarrow \infty} \sup _{\left\|\mathbf{d}_{i}\right\|_{\infty} \leq 1, E \subset \mathbb{N}} \limsup _{M \rightarrow \infty} \| \frac{1}{N} \sum_{n=1}^{N}\left(A_{x}(n) \cdot \frac{1}{M^{k}}\right.$

$$
\left.\sum_{\mathbf{m} \in[1, M]^{k}} \prod_{i=1}^{\ell} \mathbf{d}_{i, x}\left(\mathbf{m}+\left[\mathbf{b}_{i}(n)\right]\right) \cdot \mathbf{1}_{E}(n)\right) \|_{L^{2}(\mu)}^{2^{s}}
$$

is bounded by a constant $C:=C(d, k, \ell)$ times

$$
\begin{aligned}
\limsup _{H_{s} \rightarrow \infty} \frac{1}{H_{s}} \sum_{h_{s}=1}^{H_{s}} & \cdots \limsup _{H_{1} \rightarrow \infty} \frac{1}{H_{1}} \\
& \cdot \sum_{h_{1}=1}^{H_{1}} \limsup _{N \rightarrow \infty} \sup _{E \subset \mathbb{N}}\left\|\frac{1}{N} \sum_{n=1}^{N} \prod_{\boldsymbol{\epsilon} \in\{0,1\}^{s}} \mathcal{C}^{|\boldsymbol{\epsilon}|} A_{x}(n+\boldsymbol{\epsilon} \cdot \mathbf{h}) \cdot \mathbf{1}_{E}(n)\right\|_{L^{2}(\mu)},
\end{aligned}
$$

where $\left[\mathbf{b}_{i}\right]:=\left(\left[b_{i, 1}\right], \ldots,\left[b_{i, k}\right]\right)$ and $\mathbf{h}:=\left(h_{1}, \ldots, h_{s}\right)$. 
Equivalently, it suffices to prove the same estimate with the left hand side replaced with

$$
\begin{aligned}
\limsup _{N \rightarrow \infty} \limsup _{M \rightarrow \infty} \| \frac{1}{N} \sum_{n=1}^{N}\left(A_{x}(n)\right. & \cdot \frac{1}{M^{k}} \\
& \left.\cdot \sum_{\mathbf{m} \in[1, M]^{k}} \prod_{i=1}^{\ell} \mathbf{d}_{i, x, N}\left(\mathbf{m}+\left[\mathbf{b}_{i}(n)\right]\right) \cdot \mathbf{1}_{E_{N}}(n)\right) \|_{L^{2}(\mu)}^{2^{s}},
\end{aligned}
$$

where for $N \in \mathbb{N}$ the sequences of functions $\mathbf{d}_{1, N}, \ldots, \mathbf{d}_{\ell, N}: \mathbb{N}^{k} \rightarrow L^{\infty}(\mu)$ are bounded by 1 .

For $i=1, \ldots, k$, let $\mathcal{A}_{i}=\left(b_{1, i}, \ldots, b_{\ell, i}\right)$, and define the matrix type $W$ of the family of $k$-tuples $\left(\mathcal{A}_{1}, \ldots, \mathcal{A}_{k}\right)$ as in Section [5.1. Notice that having fixed $d, k, \ell$, there is only a finite number of possibilities for $W$. The proof of the claim is going to proceed by induction on $W$. We remark that it suffices to show that the constants $C$ and $s$ depend only on $W, k$, and $\ell$. Furthermore, we can assume that $b_{1,1}$ is the function with the largest growth rate.

Base case. We assume that $d=-1$, in which case all functions $b_{i, j}(t)$ converge to 0 . Then for $i=1, \ldots, \ell$, for all large enough $n \in \mathbb{N}$ the sequence $\left[\mathbf{b}_{i}\right]$ takes values on some finite subset $F_{i} \subset \mathbb{Z}^{k}$ with cardinality $\left|F_{i}\right| \leq 2^{k}$. Without loss of generality we can assume that this happens for every $n \in \mathbb{N}$. Let $E_{N, 1}, \ldots, E_{N, t}\left(t \leq 2^{k \ell}\right)$ be sets that form a partition of $S_{N}$ into sets where all the sequences $\left[b_{i, j}\right]$ are constant. Then there exist constants $\left|c_{j, N}\right| \leq 1$ such that for $s=0$ the quantity we want to estimate is equal to

$$
\begin{gathered}
\limsup _{N \rightarrow \infty}\left\|\sum_{j=1}^{t} c_{j, N}\left(\frac{1}{N} \sum_{n=1}^{N}\left(A_{x}(n) \mathbf{1}_{E_{N, j}}(n)\right)\right)\right\|_{L^{2}(\mu)} \\
\leq t \limsup _{N \rightarrow \infty} \sup _{E \subset \mathbb{N}}\left\|\frac{1}{N} \sum_{n=1}^{N}\left(A_{x}(n) \mathbf{1}_{E}(n)\right)\right\|_{L^{2}(\mu)} .
\end{gathered}
$$

Inductive step. Let $\left(\mathcal{A}_{1}, \ldots, \mathcal{A}_{k}\right)$ be a family of $\ell$ ordered $k$-tuples of functions with matrix type $W$ and degree $d \geq 0$, in which case $\operatorname{deg}\left(b_{1,1}\right) \geq 0$. Suppose that the claim holds for every family of $2 \ell$ ordered $k$-tuples of functions of matrix type $W^{\prime}$ strictly less than $W$ with $s_{0}:=s_{0}\left(W^{\prime}, k, 2 \ell\right)$ and $C:=C\left(W^{\prime}, k, 2 \ell\right)$. We let

$$
\begin{gathered}
s_{0}(W, k, \ell):=\max _{W^{\prime}<W}\left(s_{0}\left(W^{\prime}, k, 2 \ell\right)\right)+1, \\
C(W, k, \ell):=2^{(2 k \ell+1) 2^{s_{0}(W, k, \ell)-1}} \max _{W^{\prime}<W}\left(C\left(W^{\prime}, k, 2 \ell\right)\right),
\end{gathered}
$$

where the max is taken over the finitely many matrix types of families of at most $2 \ell$ functions that are smaller than $W$. The induction will be complete if we show that the asserted estimate holds for the family $\left(\mathcal{A}_{1}, \ldots, \mathcal{A}_{k}\right)$ for these values of $s(W, k, \ell)$ and $C(W, k, \ell)$. 
We start by using the Cauchy Schwarz inequality

(28)

$$
\begin{gathered}
\limsup _{N \rightarrow \infty} \limsup _{M \rightarrow \infty}\left\|\frac{1}{N} \sum_{n=1}^{N}\left(A_{x}(n) \cdot \frac{1}{M^{k}} \sum_{\mathbf{m} \in[1, M]^{k}} \prod_{i=1}^{\ell} \mathbf{d}_{i, x, N}\left(\mathbf{m}+\left[\mathbf{b}_{i}(n)\right]\right) \cdot \mathbf{1}_{E_{N}}(n)\right)\right\|_{L^{2}(\mu)}^{2} \\
\quad \leq \limsup _{N \rightarrow \infty} \limsup _{M \rightarrow \infty} \frac{1}{M^{k}} \sum_{\mathbf{m} \in[1, M]^{k}} \| \frac{1}{N} \sum_{n=1}^{N}\left(A_{x}(n)\right. \\
\left.\cdot \prod_{i=1}^{\ell} \mathbf{d}_{i, x, N}\left(\mathbf{m}+\left[\mathbf{b}_{i}(n)\right]\right) \cdot \mathbf{1}_{E_{N}}(n)\right) \|_{L^{2}(\mu)}^{2} .
\end{gathered}
$$

Using Lemma 3.6, ignoring negligible terms, and using the Cauchy-Schwarz inequality, we find that the last expression is bounded by 2 times

$$
\begin{aligned}
& \limsup _{H \rightarrow \infty} \frac{1}{H} \sum_{h=1}^{H} \limsup _{N \rightarrow \infty} \limsup _{M \rightarrow \infty} \| \frac{1}{N} \sum_{n=1}^{N}\left(A_{x}(n+h) \cdot \bar{A}_{x}(n)\right. \\
& \cdot \frac{1}{M^{k}} \sum_{\mathbf{m} \in[1, M]^{k}} \prod_{i=1}^{\ell} \mathbf{d}_{i, x, N}\left(\mathbf{m}+\left[\mathbf{b}_{i}(n+h)\right]\right) \\
&\left.\cdot \overline{\mathbf{d}}_{i, x, N}\left(\mathbf{m}+\left[\mathbf{b}_{i}(n)\right]\right) \cdot \mathbf{1}_{E_{N}}(n+h) \cdot \mathbf{1}_{E_{N}}(n)\right) \|_{L^{2}(\mu)} .
\end{aligned}
$$

We make the change of variables $\mathbf{m} \rightarrow \mathbf{m}-[\mathbf{b}(n)]$, for some vector valued function b that will be determined later. Ignoring negligible terms, we see that the last expression is equal to

(29) $\limsup _{H \rightarrow \infty} \frac{1}{H} \sum_{h=1}^{H} \limsup _{N \rightarrow \infty} \limsup _{M \rightarrow \infty} \| \frac{1}{N} \sum_{n=1}^{N}\left(A_{x}(n+h) \cdot \overline{A_{x}}(n)\right.$

$$
\begin{aligned}
\frac{1}{M^{k}} \sum_{\mathbf{m} \in[1, M]^{k}} & \prod_{i=1}^{\ell} \mathbf{d}_{i, x, N}\left(\mathbf{m}+\left[\mathbf{b}_{i}(n+h)-\mathbf{b}(n)\right]+\boldsymbol{\epsilon}_{i, h}(n)\right) \\
& \left.\cdot \overline{\mathbf{d}}_{i, x, N}\left(\mathbf{m}+\left[\mathbf{b}_{i}(n)-\mathbf{b}(n)\right]+\boldsymbol{\epsilon}_{i, h}^{\prime}(n)\right) \cdot \mathbf{1}_{E_{N, h}}(n)\right) \|_{L^{2}(\mu)},
\end{aligned}
$$

where the sequences $\left(\boldsymbol{\epsilon}_{i, h}(n)\right),\left(\boldsymbol{\epsilon}_{i, h}^{\prime}(n)\right)$ take values in $\{0,1\}^{k}$ and $E_{N, h}:=E_{N} \cap$ $\left(E_{N}-h\right)$. Notice that

$$
\begin{gathered}
\prod_{i=1}^{\ell} \mathbf{d}_{i, x, N}\left(\mathbf{m}+\left[\mathbf{b}_{i}(n+h)-\mathbf{b}(n)\right]+\boldsymbol{\epsilon}_{i, h}(n)\right) \\
\cdot \overline{\mathbf{d}}_{i, x, N}\left(\mathbf{m}+\left[\mathbf{b}_{i}(n)-\mathbf{b}(n)\right]+\boldsymbol{\epsilon}_{i, h}^{\prime}(n)\right) \cdot \mathbf{1}_{E_{N, h}}(n) \\
=\sum_{j=1}^{t} \prod_{i=1}^{\ell} \mathbf{d}_{i, j, x, N}\left(\mathbf{m}+\left[\mathbf{b}_{i}(n+h)-\mathbf{b}(n)\right]\right) \\
\cdot \mathbf{d}_{i, j, x, N}^{\prime}\left(\mathbf{m}+\left[\mathbf{b}_{i}(n)-\mathbf{b}(n)\right]\right) \cdot \mathbf{1}_{E_{N, h, j}}(n),
\end{gathered}
$$

where the sets $E_{N, h, 1}, \ldots, E_{N, h, t}\left(t \leq 2^{2 k \ell}\right)$ form a partition of $E_{N, h}$ into sets where the sequences $\boldsymbol{\epsilon}_{i}, \boldsymbol{\epsilon}_{i}^{\prime}$ are constant (either 0 or 1 ), $\mathbf{d}_{i, j, N}(\mathbf{m}):=\mathbf{d}_{i, N}\left(\mathbf{m}+c_{j}\right.$ ), and 
$\mathbf{d}_{i, j N}^{\prime}(\mathbf{m}):=\overline{\mathbf{d}}_{i, N}\left(\mathbf{m}+c_{j}^{\prime}\right)$. Combining the above we get that the limit in (29) is bounded by

$$
\begin{aligned}
& t \cdot \limsup _{H \rightarrow \infty} \frac{1}{H} \sum_{h=1}^{H} \limsup _{N \rightarrow \infty} \sup _{\left\|d_{i}\right\|_{\infty},\left\|d_{i}^{\prime}\right\|_{\infty} \leq 1, E \subset \mathbb{N}} \limsup _{M \rightarrow \infty} \| \frac{1}{N} \sum_{n=1}^{N}\left(A_{x}(n+h) \cdot \overline{A_{x}}(n)\right. \\
& \left.\cdot \frac{1}{M^{k}} \sum_{\mathbf{m} \in[1, M]^{k}} \prod_{i=1}^{\ell} \mathbf{d}_{i, x}\left(\mathbf{m}+\left[\mathbf{b}_{i}(n+h)-\mathbf{b}(n)\right]\right) \cdot \mathbf{d}_{i, x}^{\prime}\left(\mathbf{m}+\left[\mathbf{b}_{i}(n)-\mathbf{b}(n)\right]\right) \cdot \mathbf{1}_{E}(n)\right) \|_{L^{2}(\mu)} .
\end{aligned}
$$

This naturally leads us to consider a new family that consists of $2 \ell$ ordered $k$ tuples of functions. Choosing $\mathbf{b}$ exactly as in the proof of Lemma 5.5. and following the argument used there, we see that this new family has matrix type $W^{\prime}$ strictly smaller than $W$.

For this choice of $\mathbf{b}$, raising both sides of (28) to the power $2^{s\left(W^{\prime}, k, 2 \ell\right)}$, working through the previous estimates (we also use Hölder's inequality at the last step), and using the induction hypothesis, we get that for $s:=s\left(W^{\prime}, k, 2 \ell\right)+1$ and $C:=$ $C(W, k, \ell)$, defined as in (27), the left hand side in (26) is bounded by $C$ times

$$
\begin{aligned}
\limsup _{H \rightarrow \infty} & \frac{1}{H} \sum_{h=1}^{H} \limsup _{H_{s} \rightarrow \infty} \frac{1}{H_{s}} \sum_{h_{s}=1}^{H_{s}} \cdots \limsup _{H_{1} \rightarrow \infty} \frac{1}{H_{1}} \sum_{h_{1}=1}^{H_{1}} \limsup _{N \rightarrow \infty} \\
& \cdot \sup _{E \subset \mathbb{N}}\left\|\frac{1}{N} \sum_{n=1}^{N} \prod_{\boldsymbol{\epsilon} \in\{0,1\}^{s}} \mathcal{C}^{|\boldsymbol{\epsilon}|} A_{x}(n+h+\boldsymbol{\epsilon} \cdot \mathbf{h}) \cdot \mathcal{C}^{|\boldsymbol{\epsilon}|} \bar{A}_{x}(n+\boldsymbol{\epsilon} \cdot \mathbf{n}) \cdot \mathbf{1}_{E}(n)\right\|_{L^{2}(\mu)},
\end{aligned}
$$

where $\mathbf{h}=\left(h_{1}, \ldots, h_{s}\right)$. The last expression is equal to

$$
\begin{aligned}
\limsup _{H \rightarrow \infty} \frac{1}{H} \sum_{h=1}^{H} \frac{1}{H_{s}} \sum_{h_{s}=1}^{H_{s}} \cdots \limsup _{H_{1} \rightarrow \infty} \frac{1}{H_{1}} \\
\cdot \sum_{h_{1}=1}^{H_{1}} \limsup _{N \rightarrow \infty} \sup _{E \subset \mathbb{N}}\left\|\frac{1}{N} \sum_{n=1}^{N} \prod_{\boldsymbol{\epsilon} \in\{0,1\}^{s+1}} \mathcal{C}^{|\boldsymbol{\epsilon}|} A_{x}(n+\boldsymbol{\epsilon} \cdot \mathbf{h}) \cdot \mathbf{1}_{E}(n)\right\|_{L^{2}(\mu)},
\end{aligned}
$$

where $\mathbf{h}=\left(h_{1}, \ldots, h_{s}, h\right)$, as desired.

6.2. Correlation estimates for ergodic averages. Next we combine Proposition 6.1 with Proposition 5.1 in order to prove a result that will be crucial in the proof of Theorem 2.3 .

Proposition 6.2. Let $\left(X, \mathcal{X}, \mu, T_{1}, \ldots, T_{\ell}\right)$ be a system and $f_{1}, \ldots, f_{m} \in L^{\infty}(\mu)$ be functions. Let $\left(\mathcal{A}_{1}, \ldots, \mathcal{A}_{\ell}\right)$ be a nice ordered family of $\ell$-tuples of functions with degree at most $d$ and such that $\operatorname{deg}\left(a_{1,1}\right) \geq 1$. Let $\mathcal{H}$ be a Hardy field and $b_{1}, \ldots, b_{l} \in \mathcal{H}$ be functions with maximum degree $d$. Furthermore, for $i=1, \ldots, l$, let $\left(\mathcal{D}_{i}(n)\right)$ be a sequence of functions in $L^{\infty}(\mu)$, all bounded by 1 , such that for almost every $x \in X$, the sequences $\left(\mathcal{D}_{i, x}(n)\right)$ are dual sequences of level at most $r \in \mathbb{N}$. Then there exists $k=k(d, l, \ell, m, r) \in \mathbb{N}$ such that: If $\left\|f_{1}\right\|_{k, T_{1}}=0$, then the averages

$$
\frac{1}{N} \sum_{n=1}^{N} \prod_{i=1}^{m} f_{i}\left(T_{1}^{\left[a_{1, i}(n)\right]} \cdots T_{\ell}^{\left[a_{\ell, i}(n)\right]} x\right) \cdot \prod_{i=1}^{l} \mathcal{D}_{i, x}\left(\left[b_{i}(n)\right]\right)
$$

converge to 0 in $L^{2}(\mu)$. 
Proof. Let $s:=s(d, l, r)$ be as in the statement of Proposition 6.1. We assume that $\left\|f_{1}\right\|_{k, T_{1}}=0$, where $k:=k\left(d, \ell, 2^{s} \ell\right)$ is given by Proposition 5.1. We let $s^{\prime}:=2^{s}$, and for $x \in X$, let $\left(A_{x}(n)\right)$ be the sequence of $L^{\infty}(\mu)$ functions defined by

$$
A_{x}(n):=\prod_{i=1}^{m} f_{i}\left(T_{1}^{\left[a_{1, i}(n)\right]} \cdots T_{\ell}^{\left[a_{\ell, i}(n)\right]} x\right) .
$$

For $i=1, \ldots, \ell$, consider the following ordered families, each consisting of $m s^{\prime}$ functions:

$$
\mathcal{A}_{i}^{\prime}:=\left(a_{i, 1}\left(n+r_{1}\right), \ldots, a_{i, 1}\left(n+r_{s^{\prime}}\right), \ldots, a_{i, m}\left(n+r_{1}\right), \ldots, a_{i, m}\left(n+r_{s^{\prime}}\right)\right) .
$$

Since $\operatorname{deg}\left(a_{1,1}\right) \geq 1$ and $\left(\mathcal{A}_{1}, \ldots, \mathcal{A}_{\ell}\right)$ is a nice ordered family, one can check using Lemma 4.9 that $\left(\mathcal{A}_{1}^{\prime}, \ldots, \mathcal{A}_{\ell}^{\prime}\right)$ is also a nice ordered family for all $\mathbf{r}=\left(r_{1}, \ldots, r_{s^{\prime}}\right)$ in a subset $R \subset \mathbb{N}^{s^{\prime}}$ of the form

$$
R:=\left\{\mathbf{r}=\left(r_{1}, \ldots, r_{s^{\prime}}\right): r_{1} \geq c_{1}, r_{2} \geq c_{2}\left(r_{1}\right), \ldots, r_{s^{\prime}} \geq c_{s^{\prime}}\left(r_{1}, \ldots, r_{s^{\prime}-1}\right)\right\}
$$

for some sequences $c_{i}: \mathbb{N}^{i-1} \rightarrow \mathbb{N}$. Using Proposition 5.1 we have that

$$
\lim _{N \rightarrow \infty} \sup _{E \subset \mathbb{N}}\left\|\frac{1}{N} \sum_{n=1}^{N} \prod_{i=1}^{s^{\prime}} A_{x}\left(n+r_{i}\right) \cdot \mathbf{1}_{E}(n)\right\|_{L^{2}(\mu)}=0
$$

for all $\mathbf{r} \in R$. Furthermore, a similar conclusion holds if one replaces some of the sequences of functions $\left(A\left(n+r_{i}\right)\right)_{n \in \mathbb{N}}$ with their complex conjugates.

Hence, for a set of $\mathbf{h} \in \mathbb{N}^{s}$ that has a similar structure to $R$, we have

$$
\lim _{N \rightarrow \infty} \sup _{E \subset \mathbb{N}}\left\|\frac{1}{N} \sum_{n=1}^{N} \prod_{\epsilon \in\{0,1\}^{s}} \mathcal{C}^{|\boldsymbol{\epsilon}|} A_{x}(n+\boldsymbol{\epsilon} \cdot \mathbf{h}) \cdot \mathbf{1}_{E}(n)\right\|_{L^{2}(\mu)}=0 .
$$

We deduce from Proposition 6.1 that the averages (30) converge to 0 in $L^{2}(\mu)$, as desired.

\section{Seminorm estimates For the LOWER Degree iterates AND PROOF OF CONVERGENCE}

In this section we prove Theorem 2.3 . We first handle the case where all the iterates have super-linear growth, and later on use an averaging trick to handle the general case.

\subsection{Seminorm estimates in the positive degree case.}

Proposition 7.1. Let $\left(X, \mathcal{X}, \mu, T_{1}, \ldots, T_{\ell}\right)$ be a system and $f_{1}, \ldots, f_{\ell} \in L^{\infty}(\mu)$ be functions. Let $\mathcal{H}$ be a Hardy field and $a_{1}, \ldots, a_{\ell} \in \mathcal{G} \cap \mathcal{H}$ be functions with different growth rates and degree between 1 and $d$ for some $d \in \mathbb{N}$. Then there exists $k:=k(d, \ell)$ such that the following holds: If $\left\|f_{i}\right\|_{k, T_{i}}=0$ for some $i \in\{1, \ldots, \ell\}$, then the averages

$$
\frac{1}{N} \sum_{n=1}^{N} \prod_{i=1}^{\ell} T_{i}^{\left[a_{i}(n)\right]} f_{i}
$$

converge to 0 in $L^{2}(\mu)$. 
Proposition 7.1 follows from the following more general result:

Proposition 7.2. Let $\left(X, \mathcal{X}, \mu, T_{1}, \ldots, T_{\ell}\right)$ be a system and $f_{1}, \ldots, f_{\ell} \in L^{\infty}(\mu)$ be functions. Let $\mathcal{H}$ be a Hardy field and $a_{1}, \ldots, a_{\ell} \in \mathcal{G} \cap \mathcal{H}$ be functions with different growth rates and degree between 1 and $d$ for some $d \in \mathbb{N}$. Furthermore, let $b_{1}, \ldots, b_{l} \in \mathcal{H}$ have degree at most $d$. For $i=1, \ldots$, l, let $\left(\mathcal{D}_{i, x}(n)\right)_{n \in \mathbb{N}}$ be a uniformly bounded sequence of measurable functions such that, for almost every $x \in X$, the sequence $\left(\mathcal{D}_{i, x}(n)\right)_{n \in \mathbb{N}}$ is a dual sequence of level at most $r$. Then there exists $k:=k(d, l, \ell, r)$ such that the following holds: If $\left\|f_{i}\right\|_{k, T_{i}}=0$ for some $i \in\{1, \ldots, \ell\}$, then the averages

$$
\frac{1}{N} \sum_{n=1}^{N} \prod_{i=1}^{\ell} f_{i}\left(T_{i}^{\left[a_{i}(n)\right]} x\right) \cdot \prod_{i=1}^{l} \mathcal{D}_{i, x}\left(\left[b_{i}(n)\right]\right)
$$

converge to 0 in $L^{2}(\mu)$.

Proof. The proof goes by induction on the number $\ell$ of transformations. For $\ell=1$, the result follows from the case $\ell=1$ of Proposition 6.2. We take $\ell \geq 2$, assume that the result holds for $\ell-1$ transformations, and we are going to prove that it holds for $\ell$ transformations.

Without loss of generality we can assume that $a_{1}$ is the fastest growing function, and that all functions and dual sequences are bounded by 1. By Proposition 6.2. there exists $k_{0}:=k_{0}(d, l, \ell, r)$ such that, if $\left\|f_{1}\right\|_{k_{0}, T_{1}}=0$, then the averages (31) converge to 0 in $L^{1}(\mu)$. Let $k_{1}:=k(\tilde{d}, \tilde{l}, \ell-1, \tilde{r})$ be the integer that the induction hypothesis gives for $\tilde{d}:=\max \left\{d, k_{0}\right\}, \tilde{r}:=\max \left\{r, k_{0}\right\}$, and $\tilde{l}:=l+1$. Suppose that $\left\|f_{i}\right\|_{k_{1}, T_{i}}=0$ for some $i \in\{2, \ldots, \ell\}$. The induction will be complete if we show that the averages (31) converge to 0 in $L^{2}(\mu)$.

Let $\varepsilon>0$. By Proposition 3.4 we can express $f_{1}$ as $f_{1}=f_{s}+f_{u}+f_{e}$, where $f_{s}, f_{u}, f_{e} \in L^{\infty}(\mu),\left\|f_{u}\right\|_{k_{0}, T_{1}}=0,\left\|f_{e}\right\|_{L^{1}(\mu)} \leq \varepsilon$, and $f_{s}=\sum_{i=1}^{m} c_{i} f_{s, i}$, for some $m \in \mathbb{N}, c_{i} \in \mathbb{R}$, and $f_{s, i} \in L^{\infty}(\mu)$, and for almost every $x \in X$ the sequences $\left(f_{s, i}\left(T^{n} x\right)\right)_{n \in \mathbb{N}}$ are dual sequences of level at most $k_{0}$. As we explained before, when computing the limit in $L^{1}(\mu)$ of the averages (31), the contribution of the term $f_{u}$ is negligible. Furthermore, by the induction hypothesis, the same holds for the contribution of the term $f_{s, i}$, for $i=1, \ldots, m$, and as a consequence for the term $f_{s}$. It remains to handle the contribution of the term $f_{e}$. When $f_{1}$ is replaced by $f_{e}$, the $L^{1}(\mu)$ norm of the averages (31) can be bounded by a constant times

$$
\left\|\frac{1}{N} \sum_{n=1}^{N} T_{1}^{\left[a_{1}(n)\right]}\left|f_{e}\right|\right\|_{L^{1}(\mu)} \leq \frac{1}{N} \sum_{n=1}^{N}\left\|T_{1}^{\left[a_{1}(n)\right]}\left|f_{e}\right|\right\|_{L^{1}(\mu)}=\left\|f_{e}\right\|_{L^{1}(\mu)} \leq \varepsilon .
$$

Since $\varepsilon$ was arbitrary, we deduce that the averages (31) converge to 0 in $L^{1}(\mu)$, and as a consequence in $L^{2}(\mu)$ (since all functions $f_{i}$ are bounded). This completes the proof.

We also record a variant of this result that will be used later.

Proposition 7.3. Let $\left(X, \mathcal{X}, \mu, T_{1}, \ldots, T_{\ell}\right)$ be a system and $f_{1}, \ldots, f_{\ell} \in L^{\infty}(\mu)$ be functions. Let $\mathcal{H}$ be a Hardy field and $a_{1}, \ldots, a_{\ell} \in \mathcal{G} \cap \mathcal{H}$ be functions with different growth rates and degree between 1 and $d$. Then there exists $k:=k(d, \ell)$ such that 
the following holds: If $\left\|f_{i}\right\|_{k, T_{i}}=0$, for some $i \in\{1, \ldots, \ell\}$, then

$$
\lim _{R \rightarrow \infty} \limsup _{N \rightarrow \infty} \frac{1}{N} \sum_{n=1}^{N}\left\|\frac{1}{R} \sum_{r=1}^{R} \prod_{i=1}^{\ell} T_{i}^{\left[a_{i}(R n+r)\right]} f_{i}\right\|_{L^{2}(\mu)}=0 .
$$

Proof. Suppose that $a_{1}$ is the fastest growing function and all functions are bounded by 1 . Notice that for every $R \in \mathbb{N}$ we have

$$
\begin{aligned}
& \frac{1}{N} \sum_{n=1}^{N}\left\|\frac{1}{R} \sum_{r=1}^{R} \prod_{i=1}^{\ell} T_{i}^{\left[a_{i}(R n+r)\right]} f_{i}\right\|_{L^{2}(\mu)}^{2} \\
& \quad=\frac{1}{R^{2}} \sum_{1 \leq r_{1}, r_{2} \leq R} \frac{1}{N} \sum_{n=1}^{N} \int \prod_{i=1}^{\ell} T_{i}^{\left[a_{i}\left(R n+r_{1}\right)\right]} f_{i} \cdot T_{i}^{\left[a_{i}\left(R n+r_{2}\right)\right]} \bar{f}_{i} d \mu .
\end{aligned}
$$

For $r_{1} \neq r_{2}$, using Proposition 6.2 (the corresponding family of $\ell$-tuples is nice) we get that there exists $k_{0}:=k_{0}(d, \ell)$ such that if $\left\|f_{1}\right\|_{k_{0}, T_{1}}=0$, then the averages

$$
\frac{1}{N} \sum_{n=1}^{N} \prod_{i=1}^{\ell} T_{i}^{\left[a_{i}\left(R n+r_{1}\right)\right]} f_{i} \cdot T_{i}^{\left[a_{i}\left(R n+r_{2}\right)\right]} \bar{f}_{i}
$$

converge to 0 in $L^{2}(\mu)$. It is then straightforward to adapt the proof of Proposition 7.2 in order to get that there exists $k:=k(d, \ell)$ such that for $r_{1} \neq r_{2}$, if $\left\|f_{i}\right\|_{k, T_{i}}=0$, then the averages (33) converge to 0 in $L^{2}(\mu)$. We deduce that for every $R \in \mathbb{N}$ we have

$$
\limsup _{N \rightarrow \infty} \frac{1}{N} \sum_{n=1}^{N}\left\|\frac{1}{R} \sum_{r=1}^{R} \prod_{i=1}^{\ell} T_{i}^{\left[a_{i}(R n+r)\right]} f_{i}\right\|_{L^{2}(\mu)}^{2} \leq 1 / R .
$$

Taking $R \rightarrow \infty$ we deduce that (32) holds and completes the proof.

\subsection{Equidistribution on nilmanifolds.}

Proposition $7.4([15])$. Let $\mathcal{H}$ be a Hardy field and $a_{1}, \ldots, a_{\ell} \in \mathcal{G} \cap \mathcal{H}$ be functions with different growth rates and positive degree. For $i=1, \ldots, \ell$, let $X_{i}:=G_{i} / \Gamma_{i}$ be nilmanifolds, $b_{i} \in G_{i}$, and $x_{i} \in X_{i}$. Then the sequence

$$
\left(b_{1}^{\left[a_{1}(n)\right]} x_{1}, \ldots, b_{\ell}^{\left[a_{\ell}(n)\right]} x_{\ell}\right)
$$

is equidistributed on the nilmanifold $\prod_{i=1}^{\ell} \overline{\left\{b_{i}^{n} x_{i}: n \in \mathbb{N}\right\}}$.

For future use we record an identity that follows from the previous result: For all functions $F_{i} \in C\left(X_{i}\right)$ we have

$$
\lim _{N \rightarrow \infty} \frac{1}{N} \sum_{n=1}^{N} \prod_{i=1}^{\ell} F_{i}\left(b_{i}^{\left[a_{i}(n)\right]} x_{i}\right)=\prod_{i=1}^{\ell} \lim _{N \rightarrow \infty} \frac{1}{N} \sum_{n=1}^{N} F_{i}\left(b_{i}^{n} x_{i}\right) .
$$

We are also going to use another identity. Its proof is essentially contained in [15]. 
Proposition 7.5. Let $\mathcal{H}$ be a Hardy field and $a_{1}, \ldots, a_{\ell} \in \mathcal{G} \cap \mathcal{H}$ be functions with different growth rates and positive degree. For $i=1, \ldots, \ell$, let $X_{i}:=G_{i} / \Gamma_{i}$ be nilmanifolds, $b_{i} \in G_{i}, x_{i} \in X_{i}$, and $F \in C(X)$, where $X=X_{1} \times \cdots \times X_{\ell}$. Then (35)

$$
\lim _{R \rightarrow \infty} \limsup _{N \rightarrow \infty} \frac{1}{N} \sum_{n=1}^{N}\left|\frac{1}{R} \sum_{r=1}^{R} F\left(b_{1}^{\left[a_{1}(R n+r)\right]} x_{1}, \ldots, b_{\ell}^{\left[a_{\ell}(R n+r)\right]} x_{\ell}\right)-\int F d m_{\tilde{X}}\right|=0,
$$

where $\tilde{X}=\prod_{i=1}^{\ell} \overline{\left\{b_{i}^{n} x_{i}: n \in \mathbb{N}\right\}}$.

Sketch of the proof. Using a straightforward modification of the reduction argument of Section 5.2 in [15, we can reduce matters to proving the following statement: "For $i=1, \ldots, \ell$, let $X_{i}=G_{i} / \Gamma_{i}$ be nilmanifolds, with $G_{i}$ connected and simply connected, $x_{i} \in X_{i}, b_{i} \in G_{i}$ act ergodically on $X_{i}$ (meaning the sequence $\left(b_{i}^{n} x_{i}\right)$ is equidistributed in $X_{i}$ for every $\left.x_{i} \in X_{i}\right)$, and $F \in C(X)$, where $X=X_{1} \times \cdots \times X_{\ell}$. Then (35) holds with $X$ in place of $\tilde{X}$."

This was verified while proving Proposition 5.3 in [15], thus completing the proof.

\subsection{Proof of Theorem 2.3 in the positive degree case.}

Proposition 7.6. Theorem 2.3 holds when all functions $a_{1}, \ldots, a_{\ell}$ have positive degree.

Proof. We want to show that for every system $\left(X, \mathcal{B}, \mu, T_{1}, \ldots, T_{\ell}\right)$ and functions $f_{1}, \ldots, f_{\ell} \in L^{\infty}(\mu)$, we have

$$
\lim _{N \rightarrow \infty} \frac{1}{N} \sum_{n=1}^{N} \prod_{i=1}^{\ell} T_{i}^{\left[a_{i}(n)\right]} f_{i}=\prod_{i=1}^{\ell} \tilde{f}_{i}
$$

where converge is taken in $L^{2}(\mu)$ and $\tilde{f}_{i}:=\mathbb{E}\left(f_{i} \mid \mathcal{I}_{T_{i}}\right)$. By Proposition 7.2 there exists $k$ such that, if $\left\|f_{i}\right\|_{k, T_{i}}=0$ for some $i \in\{1, \ldots, \ell\}$, then the limit of the averages in (36) is 0 , where convergence takes place in $L^{2}(\mu)$ (and hence in $L^{1}(\mu)$ as well).

Let $\varepsilon>0$. By Proposition 3.4, for $i=1, \ldots, \ell$ we can write $f_{i}=f_{i, s}+f_{i, u}+f_{i, e}$, where $f_{i, s}, f_{i, u}, f_{i, e} \in L^{\infty}(\mu),\left\|f_{i, u}\right\|_{k, T_{i}}=0,\left\|f_{i, e}\right\|_{L^{1}(\mu)} \leq \varepsilon$, and $f_{i, s} \in L^{\infty}(\mu)$ are such that for almost every $x \in X$ the sequence $\left(f_{i, s}\left(T_{i}^{n} x\right)\right)$ is a $k$-step nilsequence, say $\left(\mathcal{N}_{i, x}(n)\right)$. As we explained before, when computing the limit in $L^{1}(\mu)$ of the averages in (36), the contribution of the terms $f_{i, u}$ is negligible. Furthermore, the same holds for the contribution of the terms $f_{i, e}$. This follows since for every $N \in \mathbb{N}$ the $L^{1}(\mu)$ norm of the averages in (36) is bounded by a constant multiple of

$$
\min _{i=1, \ldots, \ell} \frac{1}{N} \sum_{n=1}^{N}\left\|T_{i}^{\left[a_{i}(n)\right]}\left|f_{i}\right|\right\|_{L^{1}(\mu)}=\min _{i=1, \ldots, \ell}\left\|f_{i}\right\|_{L^{1}(\mu)} .
$$

Therefore, it remains to examine the contribution of the terms $f_{i, s}$. In this case, the average in (36) takes the form

$$
\frac{1}{N} \sum_{n=1}^{N} \prod_{i=1}^{\ell} \mathcal{N}_{i, x}\left(\left[a_{i}(n)\right]\right)
$$


Using identity (34) we get that the limit of this average is

$$
\prod_{i=1}^{\ell} \lim _{N \rightarrow \infty} \frac{1}{N} \sum_{n=1}^{N} \mathcal{N}_{i, x}(n)
$$

which in turn is equal to

$$
\prod_{i=1}^{\ell} \lim _{N \rightarrow \infty} \frac{1}{N} \sum_{n=1}^{N} f_{i, s}\left(T_{i}^{n} x\right) .
$$

For reasons explained before this is equal, up to a constant multiple of $\varepsilon$, to

$$
\prod_{i=1}^{\ell} \lim _{N \rightarrow \infty} \frac{1}{N} \sum_{n=1}^{N} f_{i}\left(T_{i}^{n} x\right)=\prod_{i=1}^{\ell} \tilde{f}_{i}
$$

Letting $\varepsilon \rightarrow 0$ completes the proof.

The proof of the next result is completely analogous to the proof of Proposition [7.6. one uses Proposition 7.3 in place of Proposition 7.2 and Proposition 7.5 in place of Proposition 7.4

Proposition 7.7. Let $\left(X, \mathcal{X}, \mu, T_{1}, \ldots, T_{\ell}\right)$ be a system and $f_{1}, \ldots, f_{\ell} \in L^{\infty}(\mu)$ be functions. Let $\mathcal{H}$ be a Hardy field and $a_{1}, \ldots, a_{\ell} \in \mathcal{G} \cap \mathcal{H}$ be functions with different growth rates and positive degree. Then

$$
\lim _{R \rightarrow \infty} \limsup _{N \rightarrow \infty} \frac{1}{N} \sum_{n=1}^{N}\left\|\frac{1}{R} \sum_{r=1}^{R} \prod_{i=1}^{\ell} T_{i}^{\left[a_{i}(R n+r)\right]} f_{i}-\prod_{i=1}^{\ell} \tilde{f}_{i}\right\|_{L^{2}(\mu)}=0,
$$

where $\tilde{f}_{i}=\mathbb{E}\left(f_{i} \mid \mathcal{I}_{T_{i}}\right)$.

\subsection{Proof of Theorem 2.3 in the general case.}

Proof of the main theorem in the general case. Without loss of generality we can assume that $a_{\ell} \prec a_{\ell-1} \prec \cdots \prec a_{1}$. If all functions $a_{1}, \ldots, a_{\ell}$ have degree 0 , then the result follows from Theorem 2.7 in [16]. If all functions $a_{1}, \ldots, a_{\ell}$ have positive degree, then the result was proved in the previous subsection. Hence, we can assume that there exists $m \in\{1, \ldots, \ell-1\}$ such that $\operatorname{deg}\left(a_{i}\right)=0$ for $i=m+1, \ldots, \ell$ and $\operatorname{deg}\left(a_{i}\right) \geq 1$ for $i=1, \ldots, m$.

It suffices to show that if $\tilde{f}_{i}=0$ for some $i \in\{1, \ldots, m\}$, then

$$
\limsup _{N \rightarrow \infty}\left\|\frac{1}{N} \sum_{n=1}^{N} \prod_{i=1}^{\ell} T_{i}^{\left[a_{i}(n)\right]} f_{i}\right\|_{L^{2}(\mu)}=0
$$

where the convergence takes place in $L^{2}(\mu)$. For every $R \in \mathbb{N}$ the limit in (37) is equal to

$$
\limsup _{N \rightarrow \infty}\left\|\frac{1}{N} \sum_{n=1}^{N} \frac{1}{R} \sum_{r=1}^{R} \prod_{i=1}^{\ell} T_{i}^{\left[a_{i}(n R+r)\right]} f_{i}\right\|_{L^{2}(\mu)} .
$$


Since the functions $a_{m+1}, \ldots, a_{\ell} \in \mathcal{H}$ have degree 0 , it is easy to see the following (one uses that the derivatives of the functions converge to 0 and the mean value theorem): for every $R \in \mathbb{N}$, for a set of $n \in \mathbb{N}$ of density 1 , we have $\left[a_{i}(n R+r)\right]=$ $\left[a_{i}(n R)\right]$ for $r=1, \ldots, R$ and $i=m+1, \ldots, \ell$. We deduce that the limit in (38) is equal to

$$
\limsup _{N \rightarrow \infty}\left\|\frac{1}{N} \sum_{n=1}^{N}\left(\prod_{i=m+1}^{\ell} T_{i}^{\left[a_{i}(n R)\right]} f_{i} \cdot \frac{1}{R} \sum_{r=1}^{R} \prod_{i=1}^{m} T_{i}^{\left[a_{i}(n R+r)\right]} f_{i}\right)\right\|_{L^{2}(\mu)} .
$$

This is bounded by a constant times

$$
\limsup _{N \rightarrow \infty} \frac{1}{N} \sum_{n=1}^{N}\left\|\frac{1}{R} \sum_{r=1}^{R} \prod_{i=1}^{m} T_{i}^{\left[a_{i}(n R+r)\right]} f_{i}\right\|_{L^{2}(\mu)} .
$$

Using Proposition 7.7 we see that the limit of this expression as $R \rightarrow \infty$ is equal to 0 . This completes the proof.

\section{REFERENCES}

[1] I. Assani, Pointwise convergence of ergodic averages along cubes, J. Anal. Math. 110 (2010), 241-269, DOI 10.1007/s11854-010-0006-3. MR2753294(2012b:37017)

[2] V. Bergelson, Weakly mixing PET, Ergodic Theory Dynam. Systems 7 (1987), no. 3, 337-349, DOI 10.1017/S0143385700004090. MR912373 (89g:28022)

[3] Vitaly Bergelson, Combinatorial and Diophantine applications of ergodic theory, Handbook of dynamical systems. Vol. 1B, Elsevier B. V., Amsterdam, 2006, pp. 745-869, DOI 10.1016/S1874-575X(06)80037-8. Appendix A by A. Leibman and Appendix B by Anthony Quas and Máté Wierdl. MR2186252 (2006j:37006)

[4] Vitaly Bergelson, Ergodic Ramsey theory: a dynamical approach to static theorems, International Congress of Mathematicians. Vol. II, Eur. Math. Soc., Zürich, 2006, pp. 1655-1678. MR2275664 (2008c:37006)

[5] Vitaly Bergelson and Inger J. Håland Knutson, Weak mixing implies weak mixing of higher orders along tempered functions, Ergodic Theory Dynam. Systems 29 (2009), no. 5, 13751416, DOI 10.1017/S0143385708000862. MR2545011 (2010i:37009)

[6] Vitaly Bergelson, Bernard Host, and Bryna Kra, Multiple recurrence and nilsequences, Invent. Math. 160 (2005), no. 2, 261-303, DOI 10.1007/s00222-004-0428-6. With an appendix by Imre Ruzsa. MR2138068 (2007i:37009)

[7] V. Bergelson and A. Leibman, Polynomial extensions of van der Waerden's and Szemerédi's theorems, J. Amer. Math. Soc. 9 (1996), no. 3, 725-753, DOI 10.1090/S0894-0347-96-00194-4. MR.1325795 (96j:11013)

[8] V. Bergelson, A. Leibman, and E. Lesigne, Intersective polynomials and the polynomial Szemerédi theorem, Adv. Math. 219 (2008), no. 1, 369-388, DOI 10.1016/j.aim.2008.05.008. MR.2435427(2009e:37004)

[9] Michael Boshernitzan, An extension of Hardy's class L of "orders of infinity", J. Analyse Math. 39 (1981), 235-255, DOI 10.1007/BF02803337. MR632463 (82m:26002)

[10] Michael D. Boshernitzan, Uniform distribution and Hardy fields, J. Anal. Math. 62 (1994), 225-240, DOI 10.1007/BF02835955. MR 1269206 (95e:11085)

[11] Michael Boshernitzan, Grigori Kolesnik, Anthony Quas, and Máté Wierdl, Ergodic averaging sequences, J. Anal. Math. 95 (2005), 63-103, DOI 10.1007/BF02791497. MR2145587 (2006b:37011)

[12] N. Bourbaki, Fonctions d'une variable réele, Chapitre V (Étude Locale des Fonctions), 2nd edition, Hermann, Paris, 1961. 
[13] Qing Chu, Multiple recurrence for two commuting transformations, Ergodic Theory Dynam. Systems 31 (2011), no. 3, 771-792, DOI 10.1017/S0143385710000258. MR2794947 (2012e:37013)

[14] Qing Chu, Nikos Frantzikinakis, and Bernard Host, Ergodic averages of commuting transformations with distinct degree polynomial iterates, Proc. Lond. Math. Soc. (3) 102 (2011), no. 5, 801-842, DOI 10.1112/plms/pdq037. MR2795725(2012e:37016)

[15] Nikos Frantzikinakis, Equidistribution of sparse sequences on nilmanifolds, J. Anal. Math. 109 (2009), 353-395, DOI 10.1007/s11854-009-0035-y. MR2585398(2011a:37020)

[16] Nikos Frantzikinakis, Multiple recurrence and convergence for Hardy sequences of polynomial growth, J. Anal. Math. 112 (2010), 79-135, DOI 10.1007/s11854-010-0026-z. MR2762998 (2012a:47027)

[17] Idris Assani, Some open problems, Ergodic theory, Contemp. Math., vol. 485, Amer. Math. Soc., Providence, RI, 2009, pp. 159-162, DOI 10.1090/conm/485/09499. MR2553216 (2010i:60001)

[18] N. Frantzikinakis, E. Lesigne, and M. Wierdl, Random sequences and pointwise convergence of multiple ergodic averages, Indiana Univ. Math. J. 61 (2012), no. 2, 585-617, DOI 10.1512/iumj.2012.61.4571. MR3043589

[19] Harry Furstenberg, Ergodic behavior of diagonal measures and a theorem of Szemerédi on arithmetic progressions, J. Analyse Math. 31 (1977), 204-256. MR0498471 (58 \#16583)

[20] H. Furstenberg, Recurrence in ergodic theory and combinatorial number theory, Princeton University Press, Princeton, N.J., 1981. M. B. Porter Lectures. MR603625 (82j:28010)

[21] H. Furstenberg and Y. Katznelson, An ergodic Szemerédi theorem for commuting transformations, J. Analyse Math. 34 (1978), 275-291 (1979), DOI 10.1007/BF02790016. MR531279 (82c:28032)

[22] W. T. Gowers, A new proof of Szemerédi's theorem, Geom. Funct. Anal. 11 (2001), no. 3, 465-588, DOI 10.1007/s00039-001-0332-9. MR.1844079(2002k:11014)

[23] Ben Green and Terence Tao, The quantitative behaviour of polynomial orbits on nilmanifolds, Ann. of Math. (2) 175 (2012), no. 2, 465-540, DOI 10.4007/annals.2012.175.2.2. MR2877065

[24] G. H. Hardy, Orders of infinity. The Infinitärcalcül of Paul du Bois-Reymond, Hafner Publishing Co., New York, 1971. Reprint of the 1910 edition; Cambridge Tracts in Mathematics and Mathematical Physics, No. 12. MR0349922 (50 \#2415)

[25] Bernard Host and Bryna Kra, Nonconventional ergodic averages and nilmanifolds, Ann. of Math. (2) 161 (2005), no. 1, 397-488, DOI 10.4007/annals.2005.161.397. MR.2150389 (2007b:37004)

[26] Bernard Host and Bryna Kra, Convergence of polynomial ergodic averages, Israel J. Math. 149 (2005), 1-19, DOI 10.1007/BF02772534. Probability in mathematics. MR 2191208 (2007c:37004)

[27] Bernard Host and Bryna Kra, Uniformity seminorms on $\ell^{\infty}$ and applications, J. Anal. Math. 108 (2009), 219-276, DOI 10.1007/s11854-009-0024-1. MR2544760 (2010j:11018)

[28] Bryna Kra, From combinatorics to ergodic theory and back again, International Congress of Mathematicians. Vol. III, Eur. Math. Soc., Zürich, 2006, pp. 57-76. MR2275670 (2007m:37014)

[29] Bryna Kra, Poincaré recurrence and number theory: thirty years later [comment on the reprint of MR0628658], Bull. Amer. Math. Soc. (N.S.) 48 (2011), no. 4, 497-501, DOI 10.1090/S0273-0979-2011-01343-X. MR2823016

[30] L. Kuipers and H. Niederreiter, Uniform distribution of sequences, Wiley-Interscience [John Wiley \& Sons], New York, 1974. Pure and Applied Mathematics. MR0419394 (54 \#7415)

[31] A. Leibman, Pointwise convergence of ergodic averages for polynomial sequences of translations on a nilmanifold, Ergodic Theory Dynam. Systems 25 (2005), no. 1, 201-213, DOI 10.1017/S0143385704000215. MR2122919 (2006j:37004)

[32] A. Leibman, Convergence of multiple ergodic averages along polynomials of several variables, Israel J. Math. 146 (2005), 303-315, DOI 10.1007/BF02773538. MR2151605 (2006c:28016)

[33] Ronnie Pavlov, Some counterexamples in topological dynamics, Ergodic Theory Dynam. Systems 28 (2008), no. 4, 1291-1322, DOI 10.1017/S0143385707000508. MR2437231 (2009k:37037)

[34] Terence Tao, Norm convergence of multiple ergodic averages for commuting transformations, Ergodic Theory Dynam. Systems $\mathbf{2 8}$ (2008), no. 2, 657-688, DOI 10.1017/S0143385708000011. MR2408398 (2009k:37012) 
[35] Miguel N. Walsh, Norm convergence of nilpotent ergodic averages, Ann. of Math. (2) 175 (2012), no. 3, 1667-1688, DOI 10.4007/annals.2012.175.3.15. MR2912715

[36] Tamar Ziegler, Universal characteristic factors and Furstenberg averages, J. Amer. Math. Soc. 20 (2007), no. 1, 53-97 (electronic), DOI 10.1090/S0894-0347-06-00532-7. MR2257397 $(2007 \mathrm{j}: 37004)$

Department of Mathematics, University of Crete, Voutes University Campus, HerAKLION 71003, GREECE

E-mail address: frantzikinakis@gmail.com 